\title{
Laplacian Integral Graphs with Maximum Degree 3
}

\author{
Steve Kirkland* \\ Department of Mathematics and Statistics \\ University of Regina \\ Regina, Saskatchewan, Canada S4S 0A2 \\ kirkland@math.uregina.ca
}

Submitted: Nov 15, 2007; Accepted: Sep 9, 2008; Published: Sep 22, 2008

Mathematics Subject Classifications: 05C50, 15A18

\begin{abstract}
A graph is said to be Laplacian integral if the spectrum of its Laplacian matrix consists entirely of integers. Using combinatorial and matrix-theoretic techniques, we identify, up to isomorphism, the 21 connected Laplacian integral graphs of maximum degree 3 on at least 6 vertices.
\end{abstract}

\section{Introduction}

Let $G$ be a graph on vertices $1, \ldots, n$. Its Laplacian matrix, $L$, can be written as $L=$ $D-A$, where $A$ is the $(0,1)$-adjacency matrix for $G$, and $D$ is the diagonal matrix such that for each $i=1, \ldots, n, d_{i i}$ is the degree of vertex $i$. There is a wealth of literature on Laplacian matrices in general, and in particular on the interplay between the structural properties of a graph and the eigenvalues of its corresponding Laplacian matrix. The survey papers [19], [20] provide useful overviews of results in the area of Laplacian spectral theory, while [1] surveys results on the algebraic connectivity of a graph $G$, which is defined as being the second smallest eigenvalue of its Laplacian matrix, and is denoted $\alpha(G)$. We note that an eigenvector of $L$ associated with $\alpha(G)$ is known as a Fiedler vector.

One of the themes that has arisen in the literature on Laplacian eigenvalues for graphs is that of Laplacian integral graphs - i.e. those graphs whose Laplacian spectrum consists entirely of integers. In particular, the papers [5], [10], [17] and [18] identify various families of Laplacian integral graphs, while [14] and [15] provide constructions for certain classes of

\footnotetext{
*Research partially supported by NSERC under grant number OGP0138251.
} 
Laplacian integral graphs. It is not difficult to see that any regular graph that is adjacency integral (i.e. all eigenvalues of its adjacency matrix are integers) is necessarily Laplacian integral as well. Further, it is observed in [15] that any complement reducible graph (i.e. a graph none of whose induced subgraphs is $P_{4}$ ) is also an example of a Laplacian integral graph. One of the interesting challenges in dealing with Laplacian integral graphs is that of describing, constructing, and understanding families of Laplacian integral graphs that are neither regular nor complement reducible. We note that some recent work of Grone and Merris [9] proceeds in that direction.

Given a graph $G$, we let $\Delta(G)$ denote its maximum degree. One of the natural lines of investigation for Laplacian integral graphs is to focus on graphs for which the maximum degree is not very large. It is straightforward to see that if $G$ is a connected graph and $\Delta(G) \leq 2$, then necessarily $G$ is either a path or a cycle. It is then readily determined that if $G$ is a connected Laplacian integral graph such that $\Delta(G) \leq 2$, then $G$ is one of the following graphs: $K_{2}, K_{1,2}, K_{3}, C_{4}$ and $C_{6}$. Here we use common graph theoretic notation (see [7], for example).

In this paper, we describe all connected Laplacian integral graphs $G$ on six or more vertices such that $\Delta(G)=3$. Generally, our approach proceeds by using combinatorial and eigenvalue information in order to narrow down the list of potential Laplacian integral graphs, and then checking the remaining few cases.

\section{Preliminaries}

For a connected graph $G$ on $n$ vertices, we denote its Laplacian spectral radius by $\lambda(G)$. It is straightforward to see that $\lambda(G) \leq 2 \Delta(G)$, with equality holding if and only if $G$ is bipartite and regular of degree $\Delta(G)$. Further, a result in [8] shows that $\Delta(G)+1 \leq \lambda(G)$, with equality holding only if $\Delta(G)=n-1$, in which case, $\lambda(G)=n$. In particular, in the case that $\Delta(G)=3$, we see that $4 \leq \lambda(G) \leq 6$. Observe that equality holds in the lower bound if and only if $n=4$, and it follows readily in that case that $G$ is a complement reducible graph. Similarly, if $\lambda(G)=n=5$, we find that $G$ is also a complement

reducible graph. Henceforth we restrict ourselves to the case that $n \geq 6, \Delta(G)=3$ and $G$ is Laplacian integral. If $\lambda(G)=6$, then necessarily $G$ is one of the eight cubic, bipartite adjacency integral graphs identified in [2], [3] and [21]. Figures 1 and 2 show these graphs. We note that in Figures 1 and 2, as elsewhere, the collection of numbers near each graph gives the corresponding Laplacian spectrum. Throughout the paper, a superscript in parentheses denotes the multiplicity of the eigenvalue.

Thus, for our purposes, it is enough to focus on the case that $\lambda(G)=5$ and $n \geq 6$. To that end, we let $\Gamma_{5}$ denote the set of connected Laplacian integral graphs on $n \geq 6$ vertices that have spectral radius 5 . Observe that for any such graph $G$, we must have $\Delta(G)=3$. If $G \in \Gamma_{5}$, then its minimum degree is either 1,2 or 3 . If the minimum degree is 1 , then 


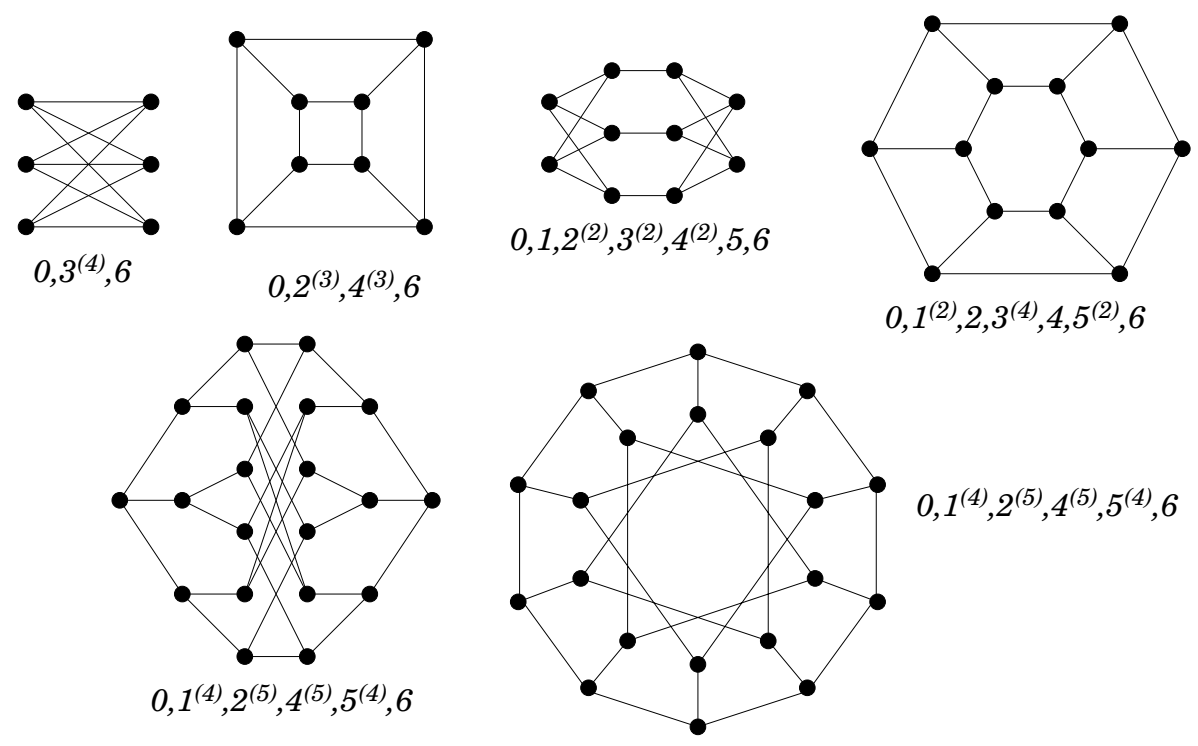

Figure 1: 3-regular, bipartite, Laplacian integral graphs

necessarily $G$ has a cutpoint. Since the algebraic connectivity of a graph is bounded above by its vertex connectivity (see [6]), and since $G$ is Laplacian integral, it then follows that $\alpha(G)=1$. A result of [12] asserts that in that case, necessarily $G$ contains a spanning star, in which case, $G$ also has $n$ as a Laplacian eigenvalue, a contradiction, since we are taking $n \geq 6$. Thus we find that the minimum degree must be at least 2 . In the case that the minimum degree is 3 , then $G$ is cubic, and necessarily adjacency integral. Referring to the results of [2], [3] and [21], we find that there are exactly four cubic connected graphs on $n \geq 6$ vertices that are Laplacian integral with Laplacian spectral radius 5 . These graphs are depicted in Figure 3. Thus it remains only to consider graphs in $\Gamma_{5}$ with minimum degree 2 and maximum degree 3 . For such a graph, we note in passing that since the number of edges of $G$ coincides with half the sum of the degrees, it follows that the parity of $n$ is the same as the parity of the number of vertices of degree 2 .

Note that for such a graph $G$, we have $\alpha(G) \leq 2$ (again by the vertex connectivity bound on $\alpha(G)$ ). Further, if $\alpha(G)=2$, we find from [16] that $G$ is a join of graphs, in which case $n$ is also an eigenvalue. As above, we find that this is impossible (since we are taking $n \geq 6>\lambda(G)=5$ ). Hence we find that necessarily $\alpha(G)$ must be 1 . According to [20], for any partition of the vertex set of $G$ as, say $A \cup B$, we have $\alpha(G) \leq \frac{n|E(A, B)|}{|A||B|}$, where $E(A, B)$ denotes the collection of edges with one endpoint in $A$ and the other endpoint in $B$. In our setting, $\alpha(G)=1$, so we find that

$$
n|E(A, B)| \geq|A||B| .
$$

In the sequel, we will frequently refer to the inequality (2.1) as following from the cut arising from the vertices in $A$ (or $B$ ). 


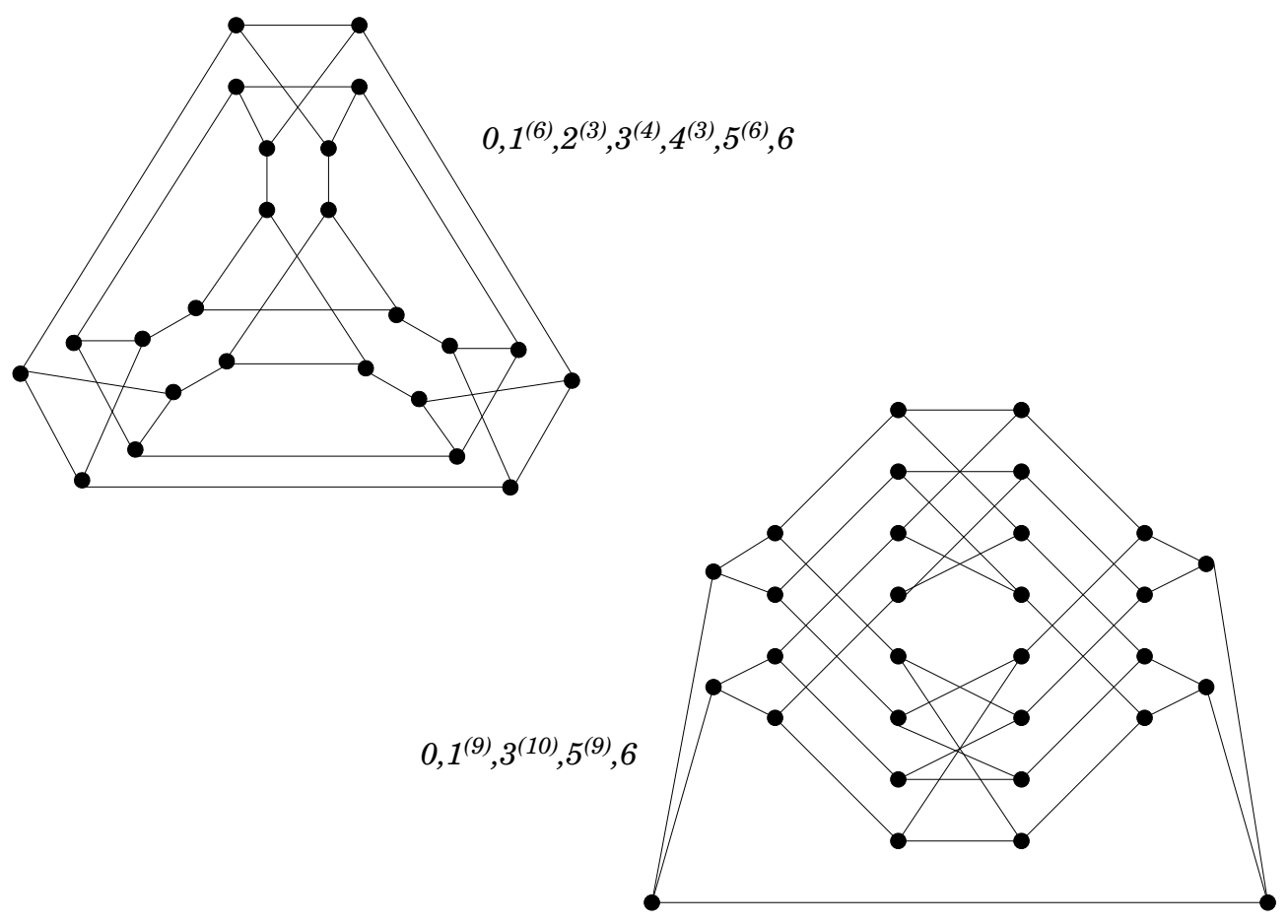

Figure 2: 3-regular, bipartite, Laplacian integral graphs

We observe also that for a graph $G \in \Gamma_{5}$ on $n$ vertices, the value of $n$ is restricted to be one of the divisors of 120 . That is because for a connected graph on $n$ vertices with distinct non-zero Laplacian eigenvalues $\lambda_{1}, \ldots, \lambda_{k}$, it follows from the Cayley-Hamilton Theorem that $\prod_{i=1}^{k}\left(L-\lambda_{i} I\right)=(-1)^{k} \frac{\prod_{i=1}^{k} \lambda_{i}}{n} J$, where $J$ denotes the all ones matrix of the appropriate order. Consequently, we see that $n$ divides $\prod_{i=1}^{k} \lambda_{i}$. In particular, for a graph on $n$ vertices in $\Gamma_{5}$, we find that $n$ divides $5 !=120$. Hence, the only admissible values of $n$ are the following: $6,8,10,12,15,20,24,30,40,60$ and 120 . For ease of notation, we let $\mathcal{G}$ denote the set of all connected graphs on $n \geq 6$ vertices with maximum degree 3 , minimum degree 2, algebraic connectivity 1 such that $n$ divides 120 . From the above considerations, we find that any non-cubic graph in $\Gamma_{5}$ is necessarily in $\mathcal{G}$.

We now state the paper's main result.

Theorem 2.1 Let $G$ be a connected non-regular graph on $n \geq 6$ vertices such that $\Delta(G)=3$. Then $G$ is Laplacian integral if and only if it is one of the graphs depicted in Figures 4 and 5 . 

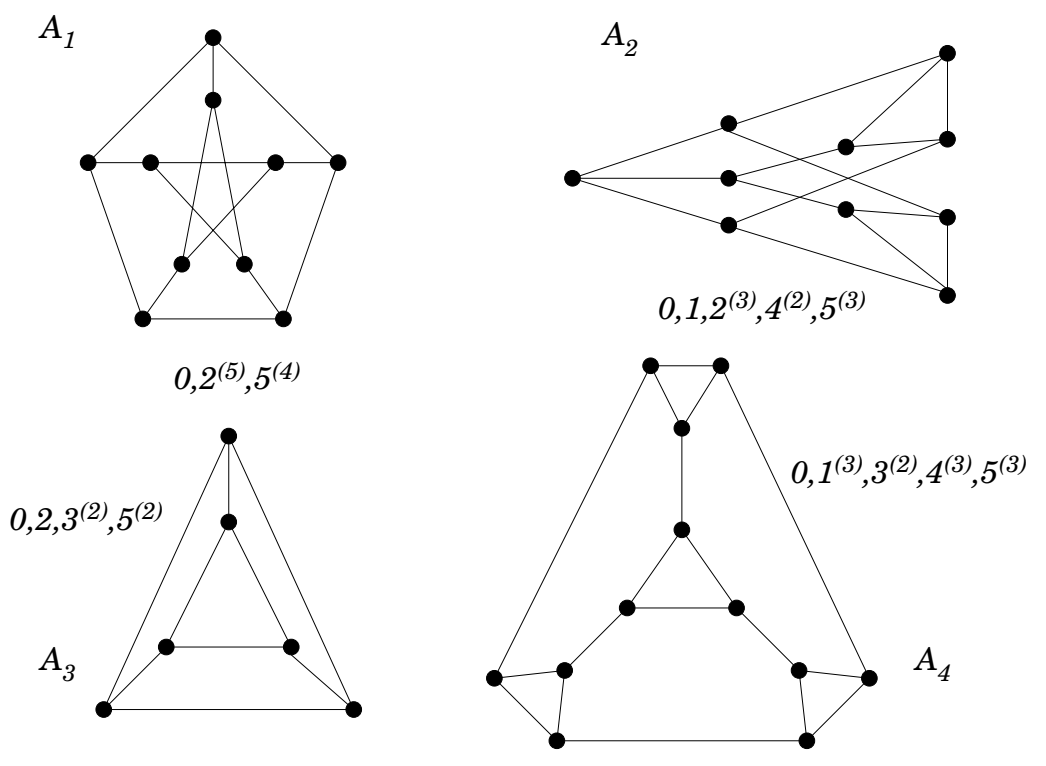

Figure 3: 3-regular, non-bipartite, Laplacian integral graphs

\section{Structural results}

In this section, we develop a number of results on the structure of graphs in $\mathcal{G}$. The following lemma will be useful in the sequel.

Lemma 3.1 Suppose that $G \in \mathcal{G}$ and has two disjoint induced subgraphs $H_{1}$ and $H_{2}$, the former on $k$ vertices and the latter on $m$ vertices, where, without loss of generality, we take $m \geq k$. Suppose further that

(i) each vertex of $H_{1} \cup H_{2}$ is adjacent to at most one vertex in $G \backslash\left(H_{1} \cup H_{2}\right)$, and

(ii) no vertex in $H_{1}$ is adjacent to any vertex in $H_{2}$.

Then necessarily each vertex in $H_{1} \cup H_{2}$ is adjacent to exactly one vertex in $G \backslash\left(H_{1} \cup H_{2}\right)$. Further, one of two cases arises:

a) we have $m=k$, and each vertex in $G \backslash\left(H_{1} \cup H_{2}\right)$, is either adjacent to no vertices in $H_{1} \cup H_{2}$, or is adjacent to precisely one vertex in $H_{1}$ and one vertex in $H_{2}$;

b) we have $m=2 k$, and each vertex in $G \backslash\left(H_{1} \cup H_{2}\right)$ is adjacent to one vertex in $H_{1}$ and two vertices in $\mathrm{H}_{2}$.

Proof: We may take the Laplacian matrix for $G$ to be written as

$$
L=\left[\begin{array}{c|c|c}
L_{1} & 0 & -X \\
\hline 0 & L_{2} & -Y \\
\hline-X^{T} & -Y^{T} & L_{3}
\end{array}\right],
$$

where the subsets of the partition correspond to $H_{1}, H_{2}$, and $G \backslash\left(H_{1} \cup H_{2}\right)$, respectively. Let 1 denote an all ones vector of the appropriate order. From the hypotheses, we find that 


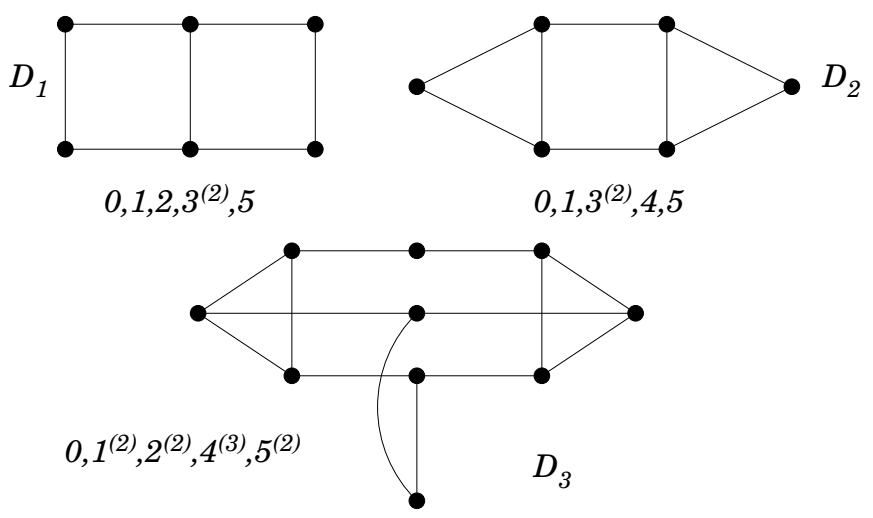

Figure 4: connected, non-regular, Laplacian integral graphs with maximum degree 3
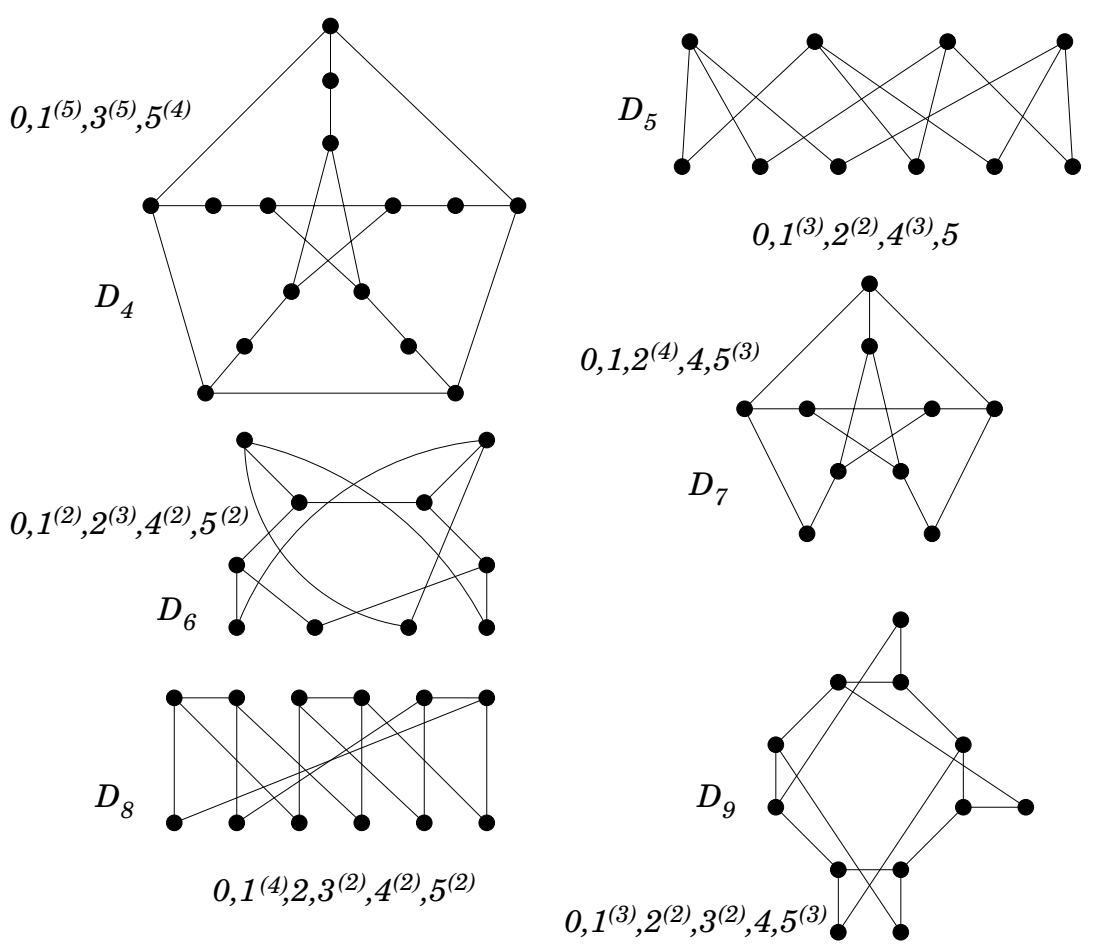

Figure 5: connected, non-regular, Laplacian integral graphs with maximum degree 3 
$0 \leq L_{1} \mathbf{1} \leq \mathbf{1}$ and $0 \leq L_{2} \mathbf{1} \leq \mathbf{1}$. Consider the vector $v=\left[\frac{m \mathbf{1}}{\frac{-k \mathbf{1}}{0}}\right]$; it is straightforward to see that $\mathbf{1}^{T} v=0$ and that $v^{T} L v \leq v^{T} v$. Note that $\alpha(G)=\min _{u^{T} \mathbf{1}=0, u^{T} u=1} u^{T} L u$, see [6]. Since $\alpha(G)=1$, it must be the case that in fact $L v=v$. It now follows that $L_{1} \mathbf{1}=\mathbf{1}, L_{2} \mathbf{1}=\mathbf{1}$, and that $m X^{T} \mathbf{1}=k Y^{T} \mathbf{1}$. The first two of those equations imply that each vertex in $H_{1} \cup H_{2}$ is adjacent to exactly one vertex in $G \backslash\left(H_{1} \cup H_{2}\right)$.

Suppose that $m=k$. Then $X^{T} \mathbf{1}=Y^{T} \mathbf{1}$, and so we see that each vertex in $G \backslash\left(H_{1} \cup H_{2}\right)$ is adjacent to the same number of vertices in $H_{1}$ as it is in $H_{2}$. Since $\Delta(G) \leq 3$, it follows that no vertex in $G \backslash\left(H_{1} \cup H_{2}\right)$ can be adjacent to two or more vertices in $H_{1}$; condition a) now follows.

Now suppose that $m>k$, and let $g=\operatorname{gcd}\{k, m\}$. Then $\frac{m}{g} X^{T} \mathbf{1}=\frac{k}{g} Y^{T} \mathbf{1}$, and since $G$ is connected, there is an index $i$ such that $e_{i}^{T} X^{T} \mathbf{1}>0$. For such an $i$, it follows that for some $p \in \mathbb{N}, e_{i}^{T} X^{T} \mathbf{1}=p \frac{k}{g}$ and $e_{i}^{T} Y^{T} \mathbf{1}=p \frac{m}{g}$. Since $p \frac{k}{g}+p \frac{m}{g} \leq 3$, we deduce that $p=1, \frac{k}{g}=1$ and $\frac{m}{g}=2$; hence $m=2 k$. In particular, a vertex corresponding to $i$ has degree 3 and is adjacent only to vertices in $H_{1} \cup H_{2}$. From the fact that $G$ is connected, we find that in fact every vertex in $G \backslash\left(H_{1} \cup H_{2}\right)$ must be adjacent to one vertex in $H_{1}$ and two vertices in $\mathrm{H}_{2}$, yielding conclusion b).

Example 3.2 The graph shown in Figure 6 illustrates conclusion a) in Lemma 3.1. Here $H_{1}$ and $H_{2}$ correspond to the subgraphs induced by the leftmost and rightmost pairs of vertices, respectively.

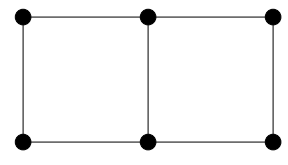

Figure 6: illustration of conclusion a) in Lemma 3.1

Example 3.3 The graph shown in Figure 7 illustrates conclusion b) in Lemma 3.1. Its Laplacian spectrum is $0,1,3-\sqrt{3}, 3^{(2)}, 3+\sqrt{3}, 4,5$. Here, $H_{1}$ consists of the subgraph induced by the leftmost pair of vertices and $H_{2}$ consists of the subgraph induced by the four rightmost vertices.

For a graph $G$ with vertices $u$ and $v$, we use the notation $u \sim v$ when $u$ is adjacent to $v$, and by a slight abuse of notation, we also use $u \sim v$ to denote the edge between those vertices.

Our approach throughout this section is to consider various subclasses of graphs in $\mathcal{G}$, and then to determine which graphs from the subclass, if any, are in $\Gamma_{5}$. We first consider 


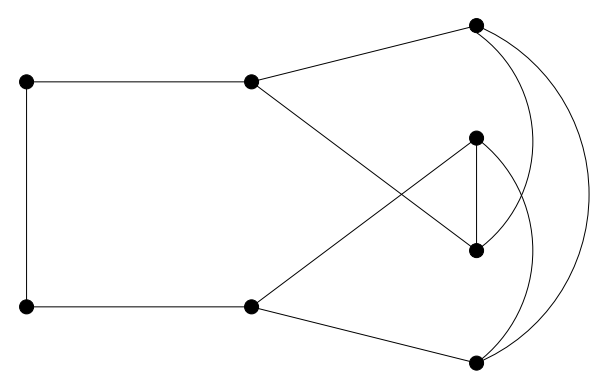

Figure 7: illustration of conclusion b) in Lemma 3.1

graphs in $\mathcal{G}$ containing at least two edges that join vertices of degree 2 (Lemmas 3.4 and 3.5). We then consider graphs in $\mathcal{G}$ according to the nature and number of 3-cycles they have (Lemmas 3.6 - 3.14 and Propositions 3.15 - 3.17). Throughout, we use $n$ to denote the number of vertices of a graph $G \in \mathcal{G}$. In general, we will think of the vertices of our graphs as being unlabeled. However, it will occasionally be useful to label vertices in some of the proofs, in order that the arguments are made more concrete.

Lemma 3.4 Suppose that $G \in \Gamma_{5}$, and that $G$ has four vertices of degree 2 labeled $1,2,3,4$, such that $1 \sim 2,3 \sim 4$. Then $G$ is isomorphic to the graph $H$ depicted in Figure 8 .

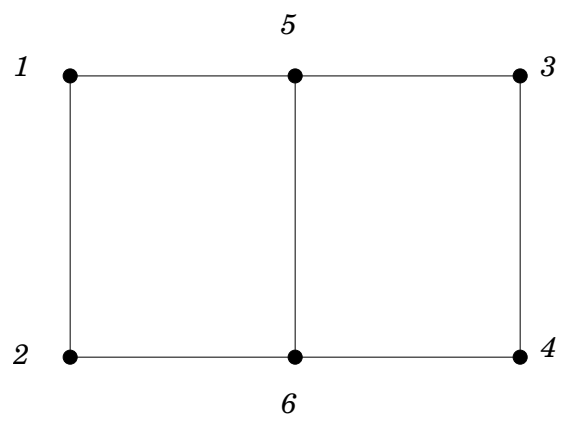

Figure 8: graph on 6 vertices from Lemma 3.4

Proof: First, suppose that neither of 1 and 2 is adjacent to either 3 or 4 . From Lemma 3.1, it follows that there are distinct vertices 5 and 6 such that, without loss of generality, 5 is adjacent to both 1 and 3 , and 6 is adjacent to both 2 and 4 . If $n=6$, we find that necessarily $G$ is the graph $H$ in Figure 8, which has Laplacian spectrum $0,1,2,3^{(2)}, 5$.

Suppose now that $n>6$. Considering the cut in $G$ arising from the vertices $1, \ldots, 6$, it follows from $(2.1)$ that $2 n \geq 6 n-36$, from which we conclude that $n \leq 9$. Referring to the list of admissible values of $n$, we find that $n$ must be 8 . It then follows that $G$ is the 


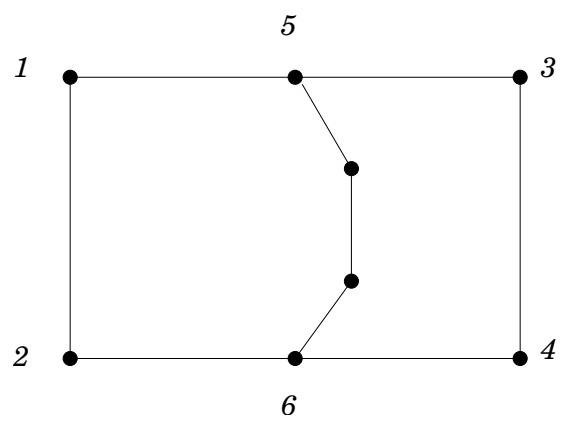

Figure 9: graph on 8 vertices from Lemma 3.4

graph depicted in Figure 9. As that graph is not Laplacian integral (here, as elsewhere, that fact is verified by a Matlab computation), we conclude that there is no Laplacian integral graph of this type on 6 or more vertices.

Next we suppose, without loss of generality, that $G$ contains the edge $2 \sim 3$. If, in addition, 1 is adjacent to 4 , then $G=C_{4}$, a contradiction. We conclude then that 1 is not adjacent to 4 . By considering the cut arising from the vertices $1, \ldots, 4$, we find that $2 n \geq 4 n-16$, so that $n$ is either 6 or 8 . For $n=6$, we are led to $G=C_{6}$, a contradiction. If $n=8$, consider the vector $x$ that is 1 on vertices $1, \ldots, 4$ and -1 on the remaining vertices. Then $x$ is orthogonal to $\mathbf{1}$, and denoting the Laplacian matrix by $L$, we have $\frac{x^{T} L x}{x^{T} x}=1$. But $x$ is not a Fiedler vector for $G$; hence $\alpha(G)<1$, a contradiction. The conclusion follows.

Lemma 3.5 Suppose that $G \in \mathcal{G}$ and that $G$ has three vertices of degree 2 that induce a $P_{3}$, but that $G$ does not have four vertices of degree $2 u_{1}, u_{2}, v_{1}, v_{2}$ such that $u_{1} \sim u_{2}$ and $v_{1} \sim v_{2}$. Then $G \notin \Gamma_{5}$.

Proof: Suppose to the contrary that $G \in \Gamma_{5}$. Let 1,2,3 denote the vertices of degree 2 that induce the $P_{3}$, say with 2 adjacent to 1 and 3 . Considering the cut arising from these three vertices, we find that $2 n \geq 3 n-9$; it follows that $n$ is 6 or 8 . If $n=6$, then $G$ has four vertices of degree 2. It now follows that $G$ must be the graph pictured in Figure 10, which is not Laplacian integral.

Suppose now that $n=8$, so that $G$ has either four or six vertices of degree 2 . If there are six vertices of degree 2, it follows that $G$ is one of the graphs in Figure 11, and hence that there must be two independent edges each of whose end points have degree 2 , contrary to our hypothesis. If there are four vertices of degree 2 , it follows that $G$ is one of the graphs depicted in Figure 12, none of which is Laplacian integral. The conclusion now follows. 


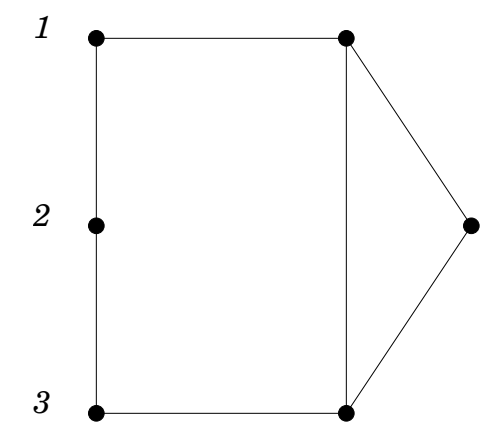

Figure 10: graph on 6 vertices from Lemma 3.5
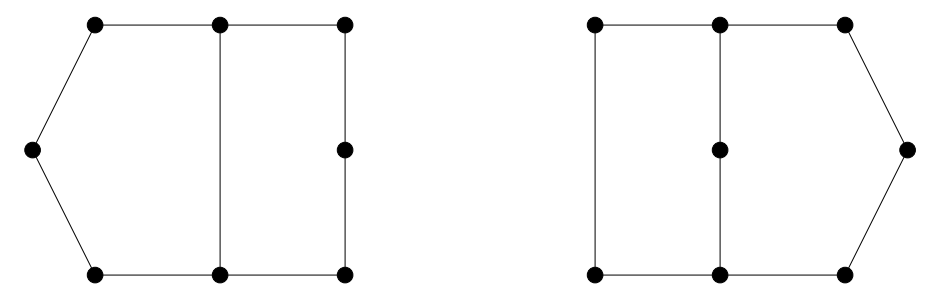

Figure 11: graphs from Lemma 3.5 with six vertices of degree 2
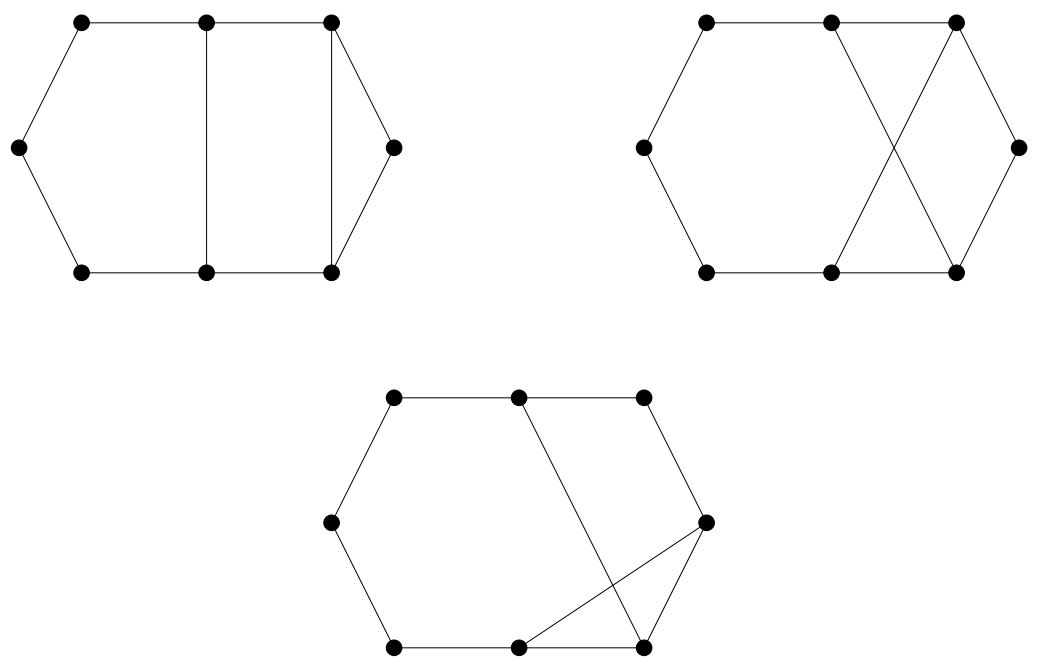

Figure 12: graphs from Lemma 3.5 with four vertices of degree 2 
In light of Lemmas 3.4 and 3.5, it suffices to consider graphs in $\mathcal{G}$ having at most one pair of adjacent vertices of degree 2. In Lemmas 3.6-3.10, we consider graphs in $\mathcal{G}$ under various hypotheses concerning their 3-cycles.

Lemma 3.6 Suppose that $G \in \mathcal{G}$ and that there are two 3-cycles in $G$ that share a vertex. Then $G \notin \Gamma_{5}$.

Proof: From the hypothesis, it follows that, without loss of generality, the induced subgraph of $G$ on vertices $1, \ldots, 4$ is as depicted in Figure 13.

Considering the cut in $G$ that arises from those vertices, we have $2 n \geq 4 n-16$, so that $n \leq 8$. We deduce that $n=6$ or $n=8$. In the former case, $G$ must be the graph depicted in Figure 14, which is not Laplacian integral.

In the latter case, it follows from Lemma 3.1 that the vector $x=\left[\frac{1}{-\mathbf{1}}\right]$ must be a Fiedler vector for $G$, but clearly that is impossible. Hence $G \notin \Gamma_{5}$.

Lemma 3.7 Suppose that $G \in \mathcal{G}$, and that $G$ has two vertex disjoint 3-cycles with no edge between any pair of vertices on the different 3 -cycles. If $G \in \Gamma_{5}$ then necessarily $G$ is isomorphic to the graph depicted in Figure 15.

Proof: Suppose that the 3-cycles are on vertices 1,2,3 and 4,5,6, respectively. From Lemma 3.1 we find that each of vertices $1, \ldots, 6$ has degree 3 , and, without loss of generality, that each of vertices 7,8 and 9 is adjacent to one of vertices 1,2,3 and one of vertices $4,5,6$. Considering the cut arising from vertices $1, \ldots, 9$, we have $3 n \geq 9 n-81$, whence either $n=10$ or $n=12$. We find that if $n=10$, then in fact $G$ is must be the graph in Figure 15, which has Laplacian spectrum $0,1^{(2)}, 2^{(2)}, 4^{(3)}, 5^{(2)}$.

If $n=12$, then considering the cut on vertices $1, \ldots, 9$, we see that there must be three edges between $\{1, \ldots, 9\}$ and $\{10,11,12\}$; we deduce then that $G$ is one of the graphs shown in Figure 16, neither of which is Laplacian integral.

Lemma 3.8 Suppose that $G \in \mathcal{G}$ and that $G$ has two vertex disjoint 3-cycles with exactly two edges joining vertices in one 3 -cycle to vertices in the other 3 -cycle. If $G \in \Gamma_{5}$, then necessarily $G$ is the graph shown in Figure 17.

Proof: It is straightforward to see that necessarily the graph in Figure 17 must be an induced subgraph of $G$. From the cut arising from that subgraph, we find that $2 n \geq 6 n-36$, so that either $n=6$ or $n=8$. If $n=6$, the conclusion is immediate, while if $n=8$, we find that $G$ must be the graph shown in Figure 18, which is not Laplacian integral. 


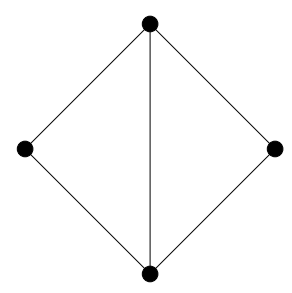

Figure 13: the induced subgraph in Lemma 3.6

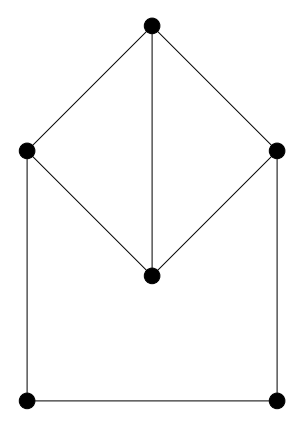

Figure 14: the graph for case that $n=6$ in Lemma 3.6

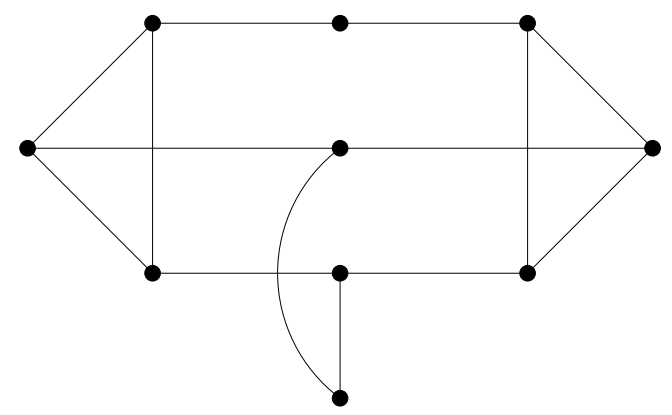

Figure 15: Laplacian integral graph from Lemma 3.7 

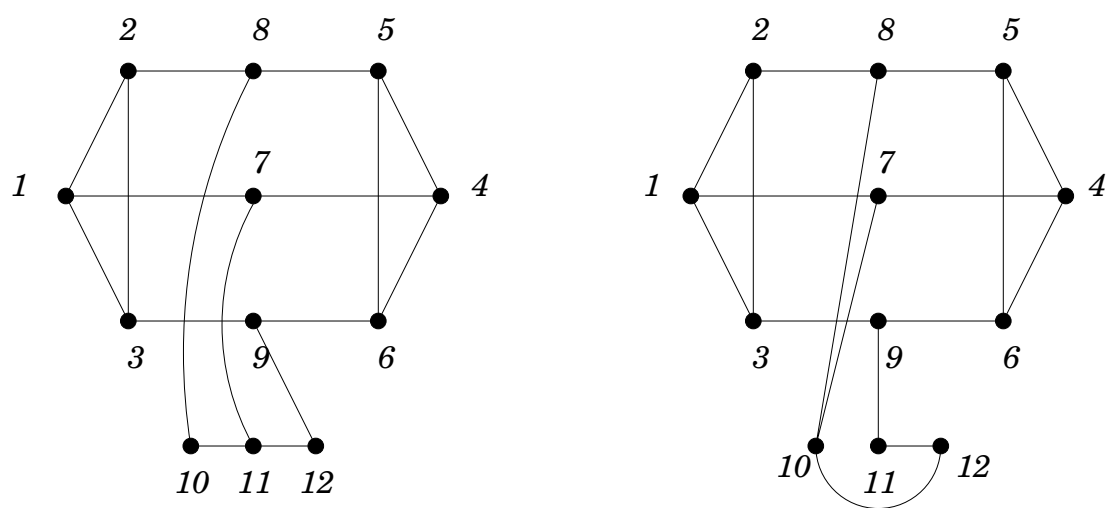

Figure 16: graphs with $n=12$ from Lemma 3.7

Lemma 3.9 Suppose that $G \in \mathcal{G}$ and that $G$ has at least three 3-cycles. Suppose further that every pair of 3-cycles is vertex disjoint, with exactly one edge between any pair of 3-cycles. Then $G \notin \Gamma_{5}$.

Proof: Observe that if $G$ has at least four 3-cycles, then from the hypotheses, $G$ must contain the graph in Figure 19 as a subgraph. As that graph is cubic, we have a contradiction.

Hence $G$ must have exactly three 3-cycles. It follows that the graph shown in Figure 20 is an induced subgraph of $G$. Considering the cut arising from the vertices of that subgraph, we have $3 n \geq 9 n-81$, from which we deduce that $n=10$ or $n=12$.

If $n=10$, it then follows that $G$ is the graph in Figure 21, which is not Laplacian integral. Suppose now that $n=12$; by considering the cut arising from the set of vertices on the three 3 -cycles, there must be at least three edges between those 9 vertices and the remaining 3 vertices. In particular, each 3-cycle consists of vertices of degree 3 . It now follows that $G$ is one of the graphs depicted in Figure 22; neither of those graphs is Laplacian integral.

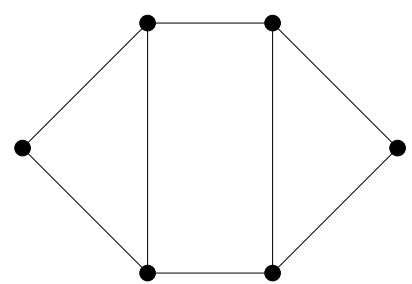

Figure 17: Laplacian integral graph from Lemma 3.8 


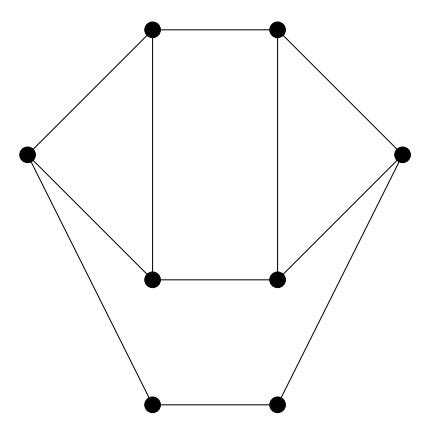

Figure 18: graph with $n=8$ in Lemma 3.8

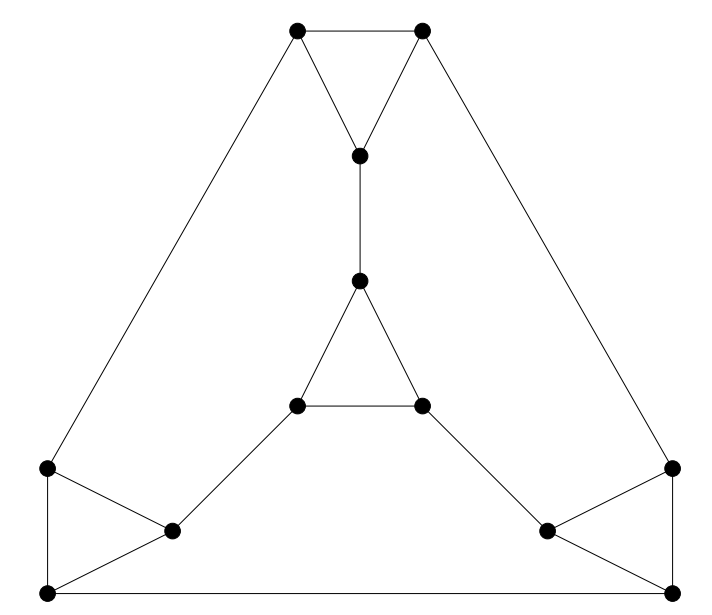

Figure 19: cubic graph in Lemma 3.9

Lemma 3.10 Suppose that $G \in \mathcal{G}$ and that $G$ has just one 3-cycle, which contains a vertex of degree 2 . Then $G \notin \Gamma_{5}$.

Proof: Considering the cut arising from the vertices of the 3 -cycle, we have $2 n \geq 3 n-9$, and we deduce the $n=6$ or $n=8$. The former leads to the graph in Figure 23, which is not Laplacian integral.

Next we consider the case $n=8$, and note that without loss of generality, necessarily $G$ must contain the subgraph shown in Figure 24. Note that either none, one, or both of vertices 4 and 5 are adjacent to two of vertices $6,7,8$. Considering the different cases leads to the six graphs in Figure 25, none of which is Laplacian integral.

Remark 3.11 Suppose that $G \in \mathcal{G}$, and that $G$ has exactly two 3-cycles, which are vertex disjoint, with exactly one edge between them. From the cut condition arising from the corresponding set of six vertices, we have $4 n \geq 6 n-36$, from which we find that $n$ is 


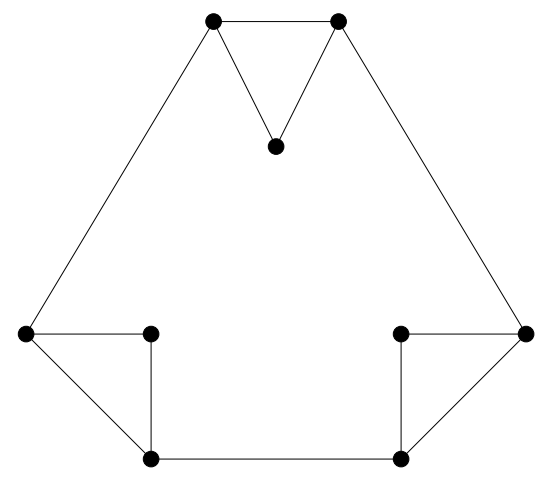

Figure 20: induced subgraph in Lemma 3.9

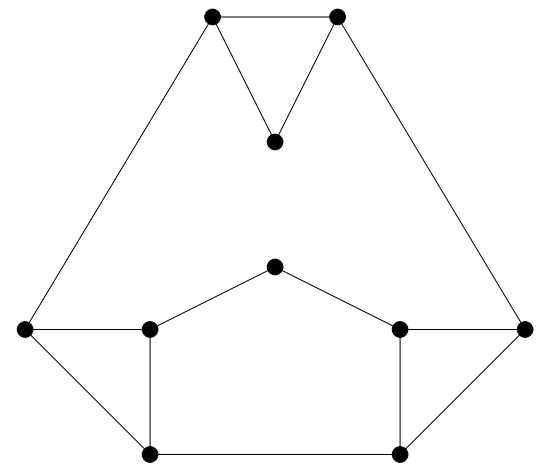

Figure 21: graph with $n=10$ in Lemma 3.9
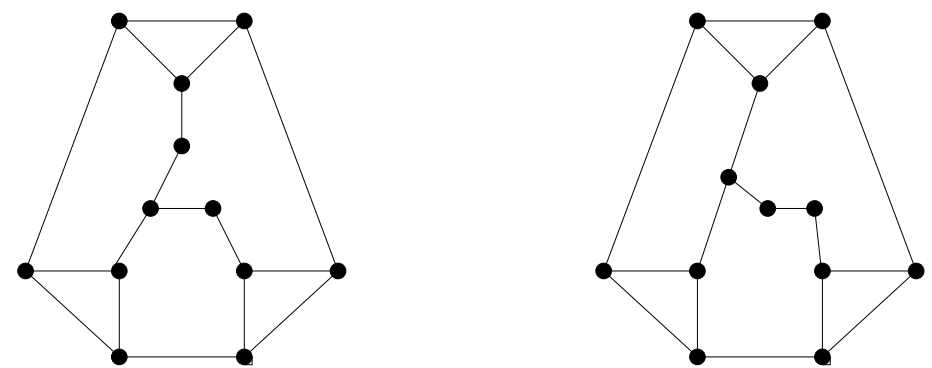

Figure 22: graphs with $n=12$ in Lemma 3.9 


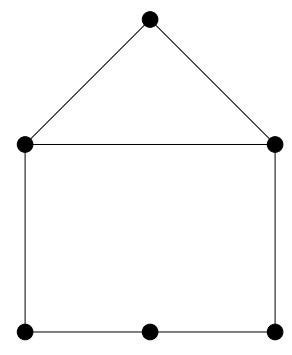

Figure 23: graph with $n=6$ in Lemma 3.10

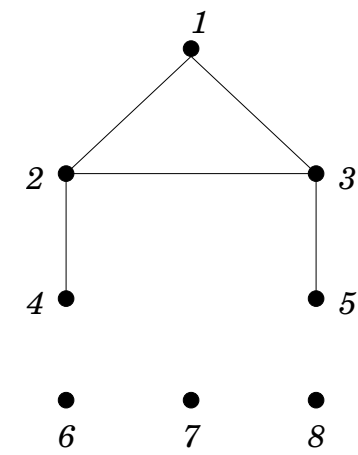

Figure 24: subgraph in Lemma 3.10
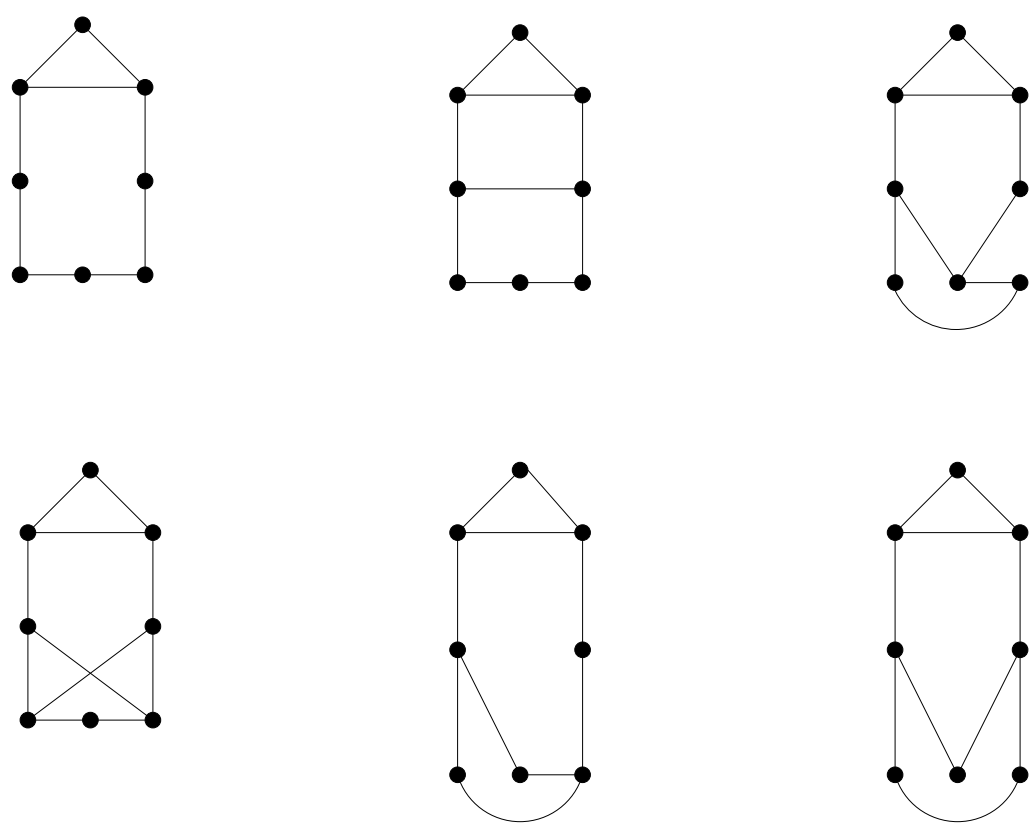

Figure 25: graphs with $n=8$ in Lemma 3.10 
one of $8,10,12$ and 15 . For the case that $n=8$, we find that $G$ must be one of the graphs shown in Figure 26, none of which is Laplacian integral.

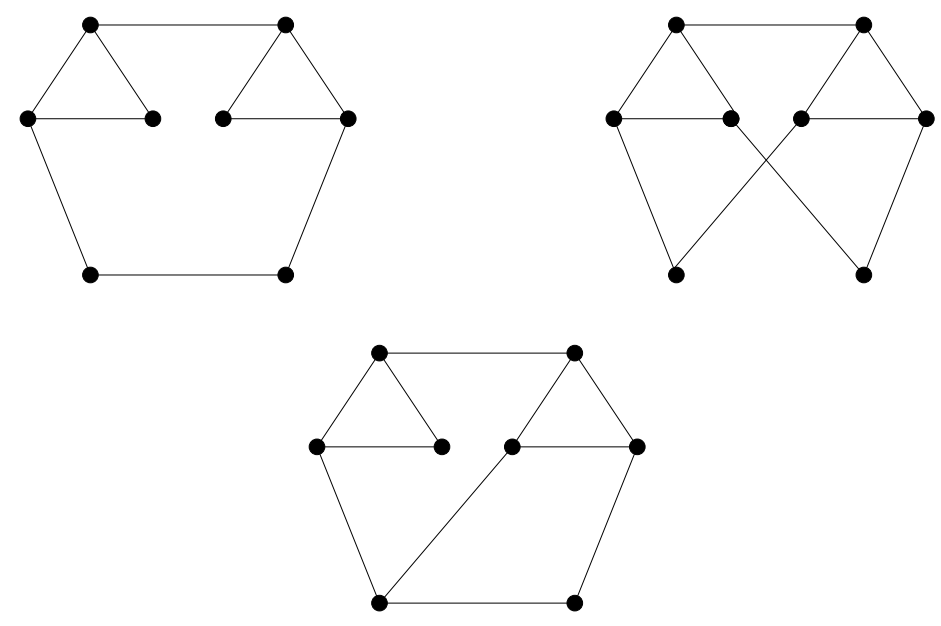

Figure 26: graphs in Remark 3.11

From Lemmas 3.6, 3.7, 3.8, 3.9, 3.10 and Remark 3.11, we find that there are three cases left to consider for a graph $G \in \mathcal{G}$ : 3-cycle-free graphs, graphs with exactly one 3cycle each of whose vertices has degree 3, and graphs on either 10, 12 or 15 vertices having exactly two 3-cycles that are vertex-disjoint, and have one edge between them. Recall that from Lemmas 3.4 and 3.5, we can also assume that there is at most one adjacent pair of vertices of degree 2 .

For any square matrix $M$, we let $\operatorname{tr}(M)$ denote its trace.

Lemma 3.12 Suppose that $G \in \mathcal{G}$ with $t 3$-cycles, a vertices of degree 2 and $n$ vertices in total. Let the Laplacian matrix of $G$ be $L$. Then

a) $\operatorname{tr}(L)=3 n-a$;

b) $\operatorname{tr}\left(L^{2}\right)=12 n-6 a$;

c) $\operatorname{tr}\left(L^{3}\right)=54 n-34 a-6 t$.

Proof: Let the degrees of the vertices in $G$ be $d_{1}, \ldots, d_{n}$. Then a) and b) follow from the facts that $\operatorname{tr}(L)=\sum_{i=1}^{n} d_{i}$ and $\operatorname{tr}\left(L^{2}\right)=\sum_{i=1}^{n}\left(d_{i}^{2}+d_{i}\right)$, respectively. Writing $L=D-A$, where $A$ is the adjacency matrix of $G$ and $D$ is the diagonal matrix of vertex degrees, we find that $\operatorname{tr}\left(L^{3}\right)=\operatorname{tr}\left(D^{3}\right)+3 \operatorname{tr}\left(D A^{2}\right)-\operatorname{tr}\left(A^{3}\right)$. Thus we have $\operatorname{tr}\left(L^{3}\right)=\sum_{i=1}^{n} d_{i}^{3}+3 \sum_{i=1}^{n} d_{i}^{2}-6 t$, and conclusion c) follows.

Lemma 3.13 Suppose that $G \in \mathcal{G}$ is on vertices labeled $1, \ldots, n$, and for each $i$, let $d_{i}$ denote the degree of vertex $i$. Suppose also that $G$ has $t 3$-cycles and a vertices of 
degree 2. Write the Laplacian matrix of $G$ as $L=D-A$, where $A$ is the adjacency matrix for $G$ and $D$ is the diagonal matrix of vertex degrees. Then $\operatorname{tr}\left(L^{4}\right)=\operatorname{tr}\left(D^{4}\right)+$ $4 \operatorname{tr}\left(A^{2} D^{2}\right)+2 \operatorname{tr}(A D A D)-4 \operatorname{tr}\left(D A^{3}\right)+\operatorname{tr}\left(A^{4}\right)$. In particular, if $G$ has $c$ 4-cycles, then $\operatorname{tr}\left(L^{4}\right)=\sum_{i=1}^{n} d_{i}^{4}+4 \sum_{i=1}^{n} d_{i}^{3}+\sum_{i=1}^{n} d_{i}^{2}+8 c+4 \sum_{i \sim j} d_{i} d_{j}+\sum_{i \sim j}\left(d_{i}+d_{j}-2\right)-4 \operatorname{tr}\left(D A^{3}\right)$.

Proof: The first conclusion is straightforward to determine. Since $\operatorname{tr}\left(D^{4}\right)=\sum_{i=1}^{n} d_{i}^{4}$ and $\operatorname{tr}\left(A^{2} D^{2}\right)=\sum_{i=1}^{n} d_{i}^{3}$, it suffices to find $\operatorname{tr}(A D A D)$ and $\operatorname{tr}\left(A^{4}\right)$. For the former expression, note that for each $i=1, \ldots, n,(A D A D)_{i i}=\sum_{j=1}^{n} e_{i}^{T} A D e_{j} e_{j}^{T} A D e_{i}=\sum_{j \sim i} d_{j} d_{i}$; it now follows that $\operatorname{tr}(A D A D)=2 \sum_{i \sim j} d_{i} d_{j}$. In order to find $\operatorname{tr}\left(A^{4}\right)$, note that for each vertex $i$, there are several different kinds of (oriented) closed walks of length 4 beginning at vertex $i$ : two consecutive 2-cycles along each edge incident with $i$, both orientations of any 4-cycle through vertex $i$, and for each vertex $j$ adjacent to $i$, a walk $i \sim j \sim k \sim j \sim i$, where $k \neq i$ is a vertex adjacent to $j$. Summing over the first type of closed walk contributes $d_{i}^{2}$ to $\operatorname{tr}\left(A^{4}\right)$, while summing over the third type contributes $\sum_{j \sim i}\left(d_{j}-1\right)$ to $\operatorname{tr}\left(A^{4}\right)$. The conclusion now follows.

Corollary 3.14 Suppose that $G \in \mathcal{G}$ with a vertices of degree 2 and $n$ vertices in total. Let the Laplacian matrix of $G$ be $L=D-A$, and suppose that $G$ has c 4-cycles.

a) If $G$ has no adjacent pairs of vertices of degree 2 , then $\operatorname{tr}\left(L^{4}\right)=258 n-192 a+8 c-$ $4 \operatorname{tr}\left(D A^{3}\right)$.

b) If $G$ has exactly one pair of adjacent vertices of degree 2 , then $\operatorname{tr}\left(L^{4}\right)=258 n-192 a+$ $8 c+4-4 \operatorname{tr}\left(D A^{3}\right)$.

Proof: Letting the degree sequence be $d_{1}, \ldots, d_{n}$, we have $\sum_{i=1}^{n} d_{i}^{4}=81 n-65 a, \sum_{i=1}^{n} d_{i}^{3}=$ $27 n-19 a, \sum_{i=1}^{n} d_{i}^{2}=9 n-5 a$, while the number of edges in $G$ is $\frac{3 n-a}{2}$.

a) Since no pairs of vertices of degree 2 are adjacent, there are $2 a$ edges of the form $i \sim j$ where $d_{i}=2$ and $d_{j}=3$, and $\frac{3 n-5 a}{2}$ edges of the form $i \sim j$ where $d_{i}=3=d_{j}=3$. We find that $\sum_{i \sim j} d_{i} d_{j}=\frac{27 n-21 a}{2}$ and $\sum_{i \sim j}\left(d_{i}+d_{j}-2\right)=6 n-4 a$, and the expression for $\operatorname{tr}\left(L^{4}\right)$ now follows from Lemma 3.13 .

b) From the hypothesis, there is exactly one edge $i \sim j$ such that $d_{i}=2=d_{j}$; hence there are $2 a-2$ edges $i \sim j$ such that $d_{i}=2$ and $d_{j}=3$ and $\frac{3 n-5 a+2}{2}$ edges $i \sim j$ with $d_{i}=3=d_{j}=3$. We then find that $\sum_{i \sim j} d_{i} d_{j}=\frac{27 n-21 a+2}{2}$ and $\sum_{i \sim j}\left(d_{i}+d_{j}-2\right)=6 n-4 a$. The expression for $\operatorname{tr}\left(L^{4}\right)$ now follows.

Proposition 3.15 Suppose that $G \in \mathcal{G}$ has $n$ vertices in total, and a vertices of degree 2. Suppose further that $G$ is 3-cycle-free, and that it has at most one pair of vertices of degree 2 that are adjacent. If $G \in \Gamma_{5}$, then it is one of the graphs shown in Figure 27. 

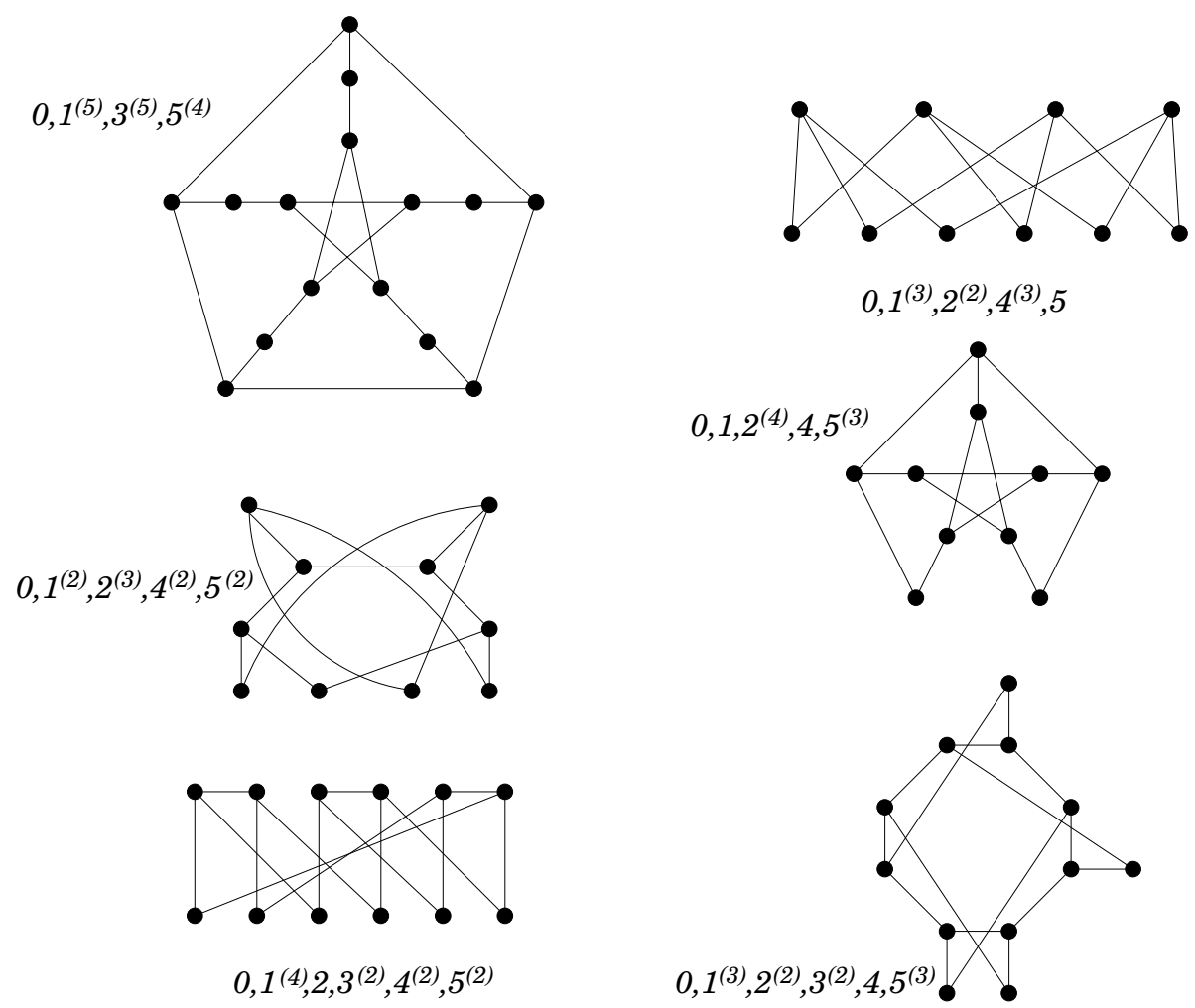

Figure 27: Laplacian integral graphs for Proposition 3.15

Proof: Suppose that $G \in \Gamma_{5}$, and let $m_{1}, \ldots, m_{5}$ denote the multiplicities of the Laplacian eigenvalues $1, \ldots, 5$, respectively (possibly one or more of the $m_{i}$ 's may be zero). Let $c$ be the number of 4 -cycles in $G$, and let $\delta$ be either 0 or 4 , according as $G$ has no pairs of adjacent vertices of degree 2 , or one pair of adjacent vertices of degree 2 , respectively. Considering $\operatorname{tr}\left(L^{k}\right), k=0, \ldots, 4$, we have, from Lemma 3.12 and Corollary 3.14, the following linear system:

$$
\left[\begin{array}{ccccc}
1 & 1 & 1 & 1 & 1 \\
1 & 2 & 3 & 4 & 5 \\
1 & 4 & 9 & 16 & 25 \\
1 & 8 & 27 & 64 & 125 \\
1 & 16 & 81 & 256 & 625
\end{array}\right]\left[\begin{array}{c}
m_{1} \\
m_{2} \\
m_{3} \\
m_{4} \\
m_{5}
\end{array}\right]=\left[\begin{array}{c}
n-1 \\
3 n-a \\
12 n-6 a \\
54 n-34 a \\
258 n-192 a+8 c+\delta
\end{array}\right] .
$$

Solving (3.2) yields

$$
\left[\begin{array}{l}
m_{1} \\
m_{2} \\
m_{3} \\
m_{4} \\
m_{5}
\end{array}\right]=n\left[\begin{array}{c}
\frac{1}{2} \\
-\frac{1}{2} \\
1 \\
-\frac{1}{2} \\
\frac{1}{2}
\end{array}\right]-a\left[\begin{array}{c}
-\frac{1}{2} \\
\frac{1}{2} \\
0 \\
-\frac{1}{2} \\
\frac{1}{2}
\end{array}\right]+c\left[\begin{array}{c}
\frac{1}{3} \\
-\frac{4}{3} \\
2 \\
-\frac{4}{3} \\
\frac{1}{3}
\end{array}\right]+\left[\begin{array}{c}
-5 \\
10 \\
-10 \\
5 \\
-1
\end{array}\right]+\delta\left[\begin{array}{c}
\frac{1}{24} \\
-\frac{1}{6} \\
\frac{1}{4} \\
-\frac{1}{6} \\
\frac{1}{24}
\end{array}\right] .
$$


If $\delta=4$, then $m_{4}+m_{5}=4-c-\frac{1}{2}$, which is not an integer, a contradiction. Thus $\delta$ must be 0 . Considering $m_{1}$, we see that $3 \mid c$. If $c>0$, then $0 \leq m_{2}+m_{4}=-n-\frac{8 c}{3}+15 \leq 7-n$, so we find that $n=6$ and $c=3$. We have $0 \leq m_{4}=-\left(\frac{n-a}{2}\right)-4+5$, so we find that $a=4$. Necessarily, each vertex of degree 2 is adjacent to both of the vertices of degree 3 , a contradiction. We conclude that $c$ must be 0 .

Thus we have $m_{1}=\frac{n+a}{2}-5, m_{2}=-\left(\frac{n+a}{2}\right)+10, m_{3}=n-10, m_{4}=-\left(\frac{n-a}{2}\right)+5$ and $m_{5}=\frac{n-a}{2}-1$. Considering $m_{3}$, we see that $n \geq 10$, while $0 \leq m_{2}=-\left(\frac{n+a}{2}\right)+10$, so that $n \leq 19$, from which we conclude that $n$ is one of 10,12 and 15 .

Next we consider the admissible values of $a$. For $n=10$ we have $a$ even, and since $1 \leq m_{5}$, we have $n-a \geq 4$, so that $a \leq 6$. Hence when $n=10, a=2,4$ or 6 . Observe that if $G \in \Gamma_{5}$, the number of edges in the subgraph induced by the vertices of degree 3 is $\frac{3 n-5 a}{2}$, so that necessarily $a \leq \frac{3 n}{5}$. For $n=12$, this yields $a \leq \frac{36}{5}$, so that $a=2,4$ or 6 . Note further that when $n=12$ and $a=2$, we have $m_{4}=0$ and $m_{1}, m_{2}, m_{3}, m_{5} \geq 1$. If $G \in \Gamma_{5}$, then 12 must divide $(2 \cdot 3 \cdot 5)$, a contradiction. Hence if $n=12, a$ is either 4 or 6 . If $n=15$, we have $0 \leq m_{4}=-\left(\frac{n-a}{2}\right)+5$, so that $a \geq n-10=5$. Also, $0 \leq m_{2}=-\left(\frac{n+a}{2}\right)+10$, so that $a \leq 20-n=5$. Hence for $n=15$, we have $a=5$.

Let $H$ be the subgraph of $G$ induced by the vertices of degree 3. Then $H$ has $n-a$ vertices and $\frac{3 n-5 a}{2}$ edges. Note that the smallest eigenvalue of the adjacency matrix of $H$, $\lambda_{\min }(H)$, say, satisfies $\lambda_{\min }(H) \geq-2$, since the Laplacian spectral radius of $G$ is 5 .

Let the Laplacian matrix of $G$ be $L=D-A$. It is straightforward to determine that $\operatorname{tr}\left(L^{5}\right)=\operatorname{tr}\left(D^{5}\right)+5 \operatorname{tr}\left(D^{3} A^{2}\right)+5 \operatorname{tr}(D A D A D)+5 \operatorname{tr}\left(D A^{4}\right)-\operatorname{tr}\left(A^{5}\right)$. Note that since $G$ has no 4-cycles, $\operatorname{tr}\left(D A^{4}\right)=\sum_{i=1}^{n} d_{i}^{3}+\sum_{i=1}^{n} \sum_{j \sim i} d_{i}\left(d_{j}-1\right)=\sum_{i=1}^{n} d_{i}^{3}+2 \sum_{i \sim j} d_{i} d_{j}-\sum_{i=1}^{n} d_{i}^{2}=$ $45 n-35 a$. Also, $\operatorname{tr}(D A D A D)=\sum_{i=1}^{n} d_{i}\left(\sum_{j=1}^{n} e_{i}^{T} A D e_{j} e_{j}^{T} A D e_{i}\right)=$

$\sum_{i=1}^{n} d_{i} \sum_{j \sim i} d_{i} d_{j}=\sum_{i \sim j} d_{i} d_{j}\left(d_{i}+d_{j}\right)=81 n-75 a$. Letting $w_{5}$ denote the number of 5 cycles in $G$, it now follows that $\operatorname{tr}\left(L^{5}\right)=1278 n-1086 a-10 w_{5}$. Considering the expressions for $m_{1}, \ldots, m_{5}$, we also find that $\operatorname{tr}\left(L^{5}\right)=1278 n-1066 a-120$, from which we conclude $w_{5}=12-2 a$.

Suppose first that $n=15$ and $a=5$. Then $m_{2}=0=m_{4}$, and it follows from the Cayley-Hamilton Theorem that $L^{3}-9 L^{2}+23 L-15 I=-J$, or equivalently, that

$$
\begin{array}{r}
15 I-J=D^{3}-A D^{2}-D A D-D^{2} A+A^{2} D+A D A+D A^{2}-A^{3}-9 D^{2}+9 A D \\
+9 D A-9 A^{2}+23 D-23 A .
\end{array}
$$

Considering a diagonal entry corresponding to a vertex $i$ of degree 3 , we have $27+9+$ $\sum_{j \sim i} d_{j}+9-81-27+69=14$, so that $\sum_{j \sim i} d_{j}=8$. We deduce that each vertex of degree 3 is adjacent to one vertex of degree 2 . Now considering vertices $i, j$ of degree 2 (necessarily adjacent only to vertices of degree 3) we find that $2 e_{i}^{T} A^{2} e_{j}+3 e_{i}^{T} A^{2} e_{j}+$ $2 e_{i}^{T} A^{2} e_{j}-e_{i}^{T} A^{3} e_{j}-9 e_{i}^{T} A^{2} e_{j}=-1$, or equivalently, $2 e_{i}^{T} A^{2} e_{j}+e_{i}^{T} A^{3} e_{j}=1$. We conclude that $i$ and $j$ have no common neighbours (otherwise $e_{i}^{T} A^{2} e_{j} \geq 1$ ) and that there is exactly one path of length 3 between vertices $i$ and $j$. Consider the subgraph $H$, which has 10 
vertices and 10 edges. Since each vertex in $G$ of degree 3 is adjacent to one vertex of degree 2, we find that $H$ is regular of degree 2. Thus, $H$ is either a single cycle (necessarily a $C_{10}$ ), or a union of cycles.

Suppose first that $H=C_{10}$. Consider two vertices of $i, j$ at distance 2 in $H$. From the facts that $d_{i}=d_{j}=3$ and $A_{i, j}=0$, we find from (3.4) that $-3 e_{i}^{T} A^{2} e_{j}+e_{i}^{T} A D A e_{j}-$ $e_{i}^{T} A^{3} e_{j}=-1$. Observe that there is just one path of length 2 between $i$ and $j$ (two or more such paths would lead to a $C_{4}$, a contradiction), so that in fact $e_{i}^{T} A^{2} e_{j}=1$ and $e_{i}^{T} A D A e_{j}=3$; we conclude that $e_{i}^{T} A^{3} e_{j}=1$, i.e. there is also path of length 3 from $i$ to $j$. Thus, any two vertices at distance 2 in $H$ are on a common cycle of length 5 . It now follows that $G$ has more than two cycles of length 5 , a contradiction. We conclude that $H \neq C_{10}$.

Hence, $H$ must be a union of cycles, and since the girth of $G$ is 5 , it follows that in fact $H$ is a disjoint union of two 5 -cycles. Using the fact that there is one path of length 3 between any pair of vertices of degree 2 , it follows that $G$ is the graph pictured in Figure 28, which has Laplacian spectrum $0,1^{(5)}, 3^{(5)}, 5^{(4)}$.

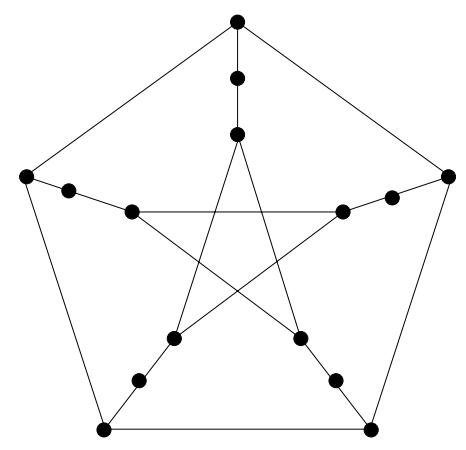

Figure 28: Laplacian integral graph of Proposition 3.15 with $n=15, a=5$

Next, we consider the case that $n=10$. If $a=6$, then $3 n-5 a=0$, so $H$ is the empty graph on 4 vertices, and $w_{5}=12-2 a=0$. It turns out that there is just one graph satisfying all of the necessary conditions; it is pictured in Figure 29, and has Laplacian spectrum $0,1^{(3)}, 2^{(2)}, 4^{(3)}, 5$.

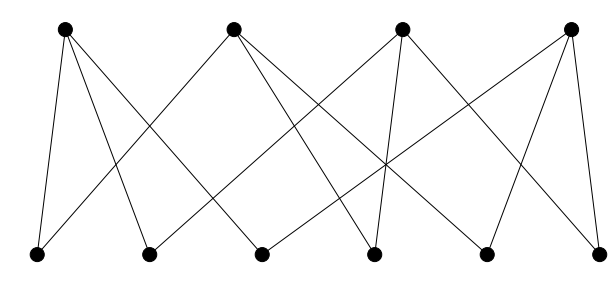

Figure 29: Laplacian integral graph of Proposition 3.15 with $n=10, a=6$ 
If $n=10$ and $a=4$, then $H$ has 6 vertices and 5 edges, and $w_{5}=4$. If $H$ is disconnected, it follows that necessarily $H=C_{5} \cup K_{1}$, but then necessarily one vertex of degree 2 is adjacent to two vertices on the $C_{5}$, a contradiction to the fact that the girth of $G$ is 5 . Thus, $H$ is connected, and is necessarily a tree whose adjacency spectral radius is at most 2. It follows that $H$ is one of the graphs pictured in Figure 30.

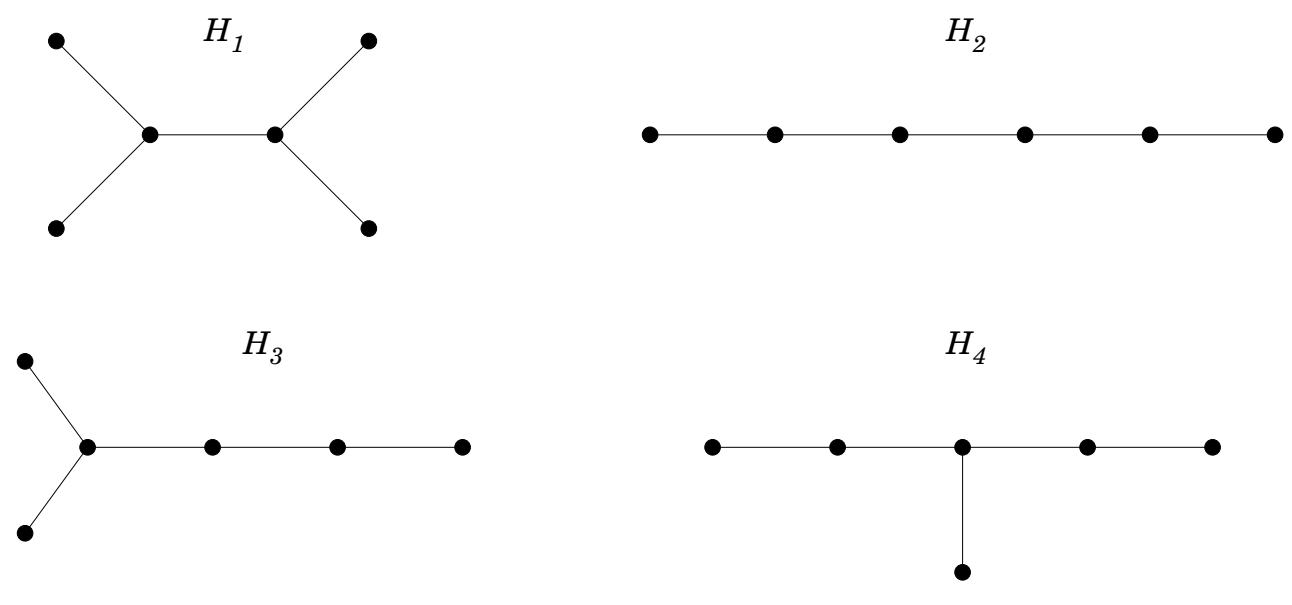

Figure 30: choices for $H$ when $n=10, a=4$ in Proposition 3.15

There is just one possible graph $G$ such that $H_{1}=H$; it is pictured in Figure 31 and has Laplacian spectrum $0,1^{(2)}, 2^{(3)}, 4^{(2)}, 5^{(2)}$.

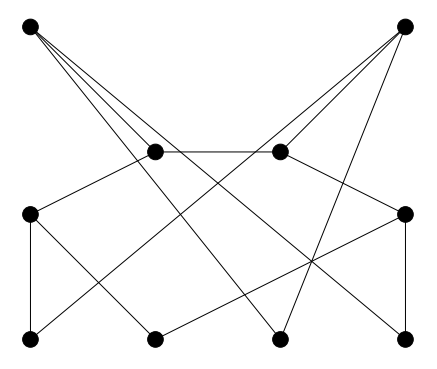

Figure 31: Laplacian integral graph for $n=10, a=4$ in Proposition 3.15

If $H=H_{2}$, there are just two candidate graphs for $G$. These are shown in Figure 32, and neither is Laplacian integral.

If $H=H_{3}$, it follows that $G$ must contain the subgraph pictured in Figure 33; attempting to complete that subgraph to a graph with the required degree sequence, we see that there is no $C_{4}$-free completion.

Finally, if $H=H_{4}$, there is only one admissible candidate for $G$, shown in Figure 34; it is not Laplacian integral. 

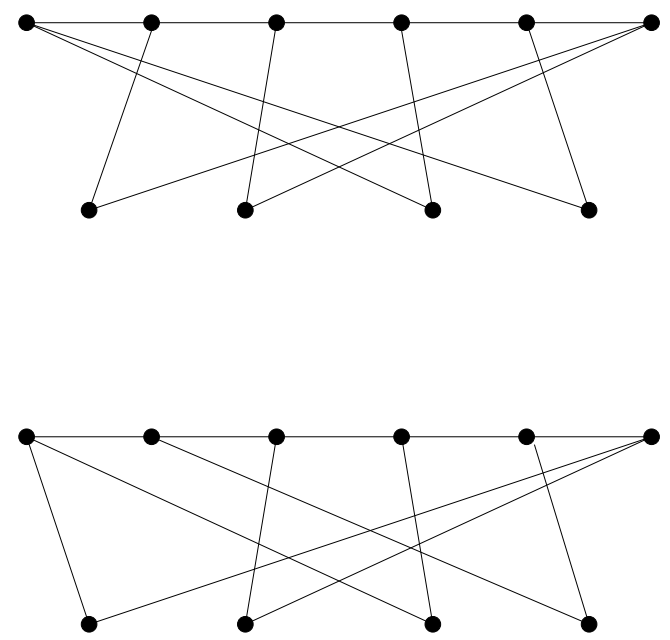

Figure 32: graphs arising from $H_{2}$ in Proposition 3.15

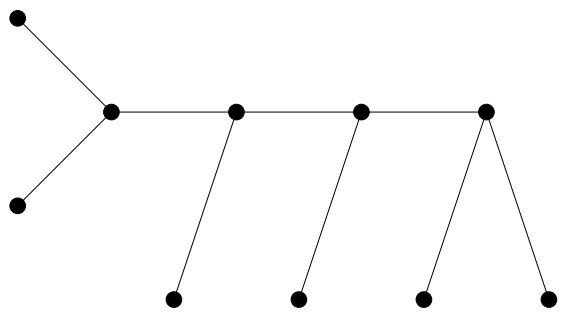

Figure 33: subgraph arising from $H_{3}$ in Proposition 3.15

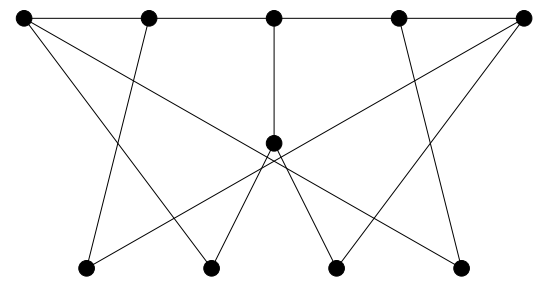

Figure 34: graph arising from $H_{4}$ in Proposition 3.15 
Next, we consider the case that $n=10$ and $a=2$. Then $H$ has 8 vertices and 10 edges, and $w_{5}=8$. We suppose for concreteness that the vertices of $H$ are labeled $1, \ldots, 8$. If $H$ is bipartite, then each connected component is either a cycle or a tree, and hence $H$ has at most 8 edges, a contradiction. Thus, $H$ must contain either a $C_{5}$ or a $C_{7}$. It is readily seen that if $H$ contains a $C_{7}$ then it must also contain a $C_{3}$ or $C_{4}$, a contradiction. Hence $H$ must contain a $C_{5}$, say on vertices $1, \ldots, 5$. Each of vertices $6,7,8$ is adjacent to at most one of vertices $1, \ldots, 5$, and there are at most two edges between vertices 6,7 , 8 , so it follows that each of $6,7,8$ is adjacent to exactly one vertex in $\{1, \ldots, 5\}$. Without loss of generality, we have the following subgraph of $H$, pictured in Figure 35.

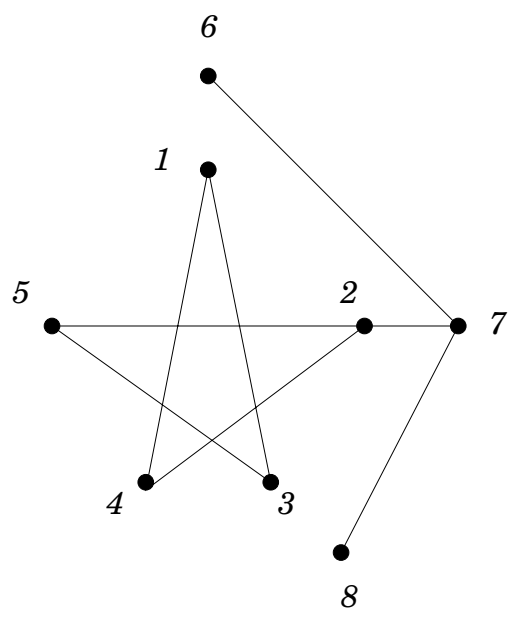

Figure 35: subgraph of $H$ when $n=10, a=2$ in Proposition 3.15

If $6 \sim 5$, or $6 \sim 4$, then $G$ contains a $C_{4}$, a contradiction; hence without loss of generality, $6 \sim 1$. There is only one way to complete the graph $G$ so as to maintain the degree sequence and avoid a $C_{4}$, and that completion yields the graph shown in Figure 36 , which has Laplacian spectrum $0,1,2^{(4)}, 4,5^{(3)}$.

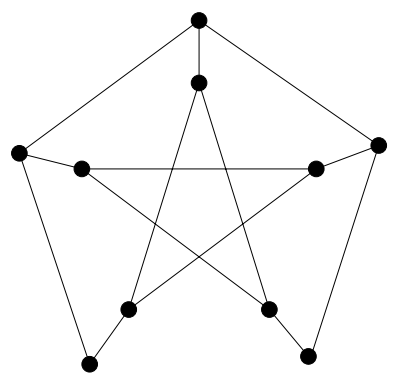

Figure 36: Laplacian integral graph for $n=10, a=2$ 
Next we consider the case that $n=12$. Suppose first that $a=6$, so that $H$ has 3 edges, and 6 vertices and $w_{5}=0$. If $H=K_{1,3} \cup \overline{K_{2}}$, there is just one $C_{4}$-free completion with the required degree sequence, shown in Figure 37; that graph is not Laplacian integral.

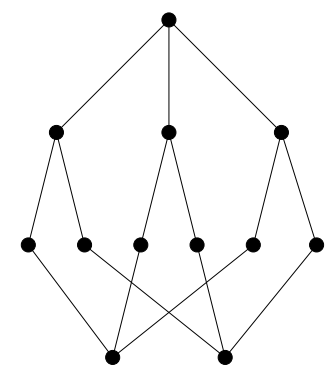

Figure 37: graph arising when $H=K_{1,3} \cup \overline{K_{2}}$

If $H=P_{3} \cup K_{2} \cup K_{1}$, we obtain the graph in Figure 38 as the only admissible candidate for $G$, and that graph is not Laplacian integral.

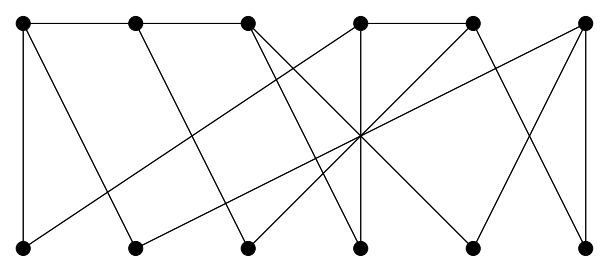

Figure 38: graph in Proposition 3.15 arising from $H=P_{3} \cup K_{2} \cup K_{1}$

If $H=K_{2} \cup K_{2} \cup K_{2}$, we find that there are only two admissible completions, both are shown in Figure 39. The graph $G_{1}$ is not Laplacian integral, but the graph $G_{2}$ is, with Laplacian spectrum $0,1^{(4)}, 2,3^{(2)}, 4^{(2)}, 5^{(2)}$.

Lastly, we consider the case $n=12, a=4$. Note that $H$ has 8 vertices and 8 edges, and that $w_{5}=4$. If $H$ is bipartite, it follows that necessarily $H=C_{8}$ (recall that necessarily $\lambda_{\min }(H) \geq-2$ ). Since $G$ is not bipartite, there are just two admissible completions, both are shown in Figure 40. Note that $G_{1}$ has Laplacian spectrum $0,1^{(3)}, 2^{(2)}, 3^{(2)}, 4,5^{(3)}$, while $G_{2}$ is not Laplacian integral.

Next, suppose that $H$ has an odd cycle. If $H$ contains a $C_{7}$, then necessarily we find that $H$ consists of a 7-cycle with a pendant vertex and edge attached. That graph can be completed to one of three admissible graphs; these are shown in Figure 41, and none is Laplacian integral.

Suppose now that $H$ contains a $C_{5}$. Noting that the graphs in Figure 42 have smallest adjacency eigenvalue less than -2 , it follows that $H$ is one of the graphs $H_{5}, H_{6}, H_{7}$ shown in Figure 43. 

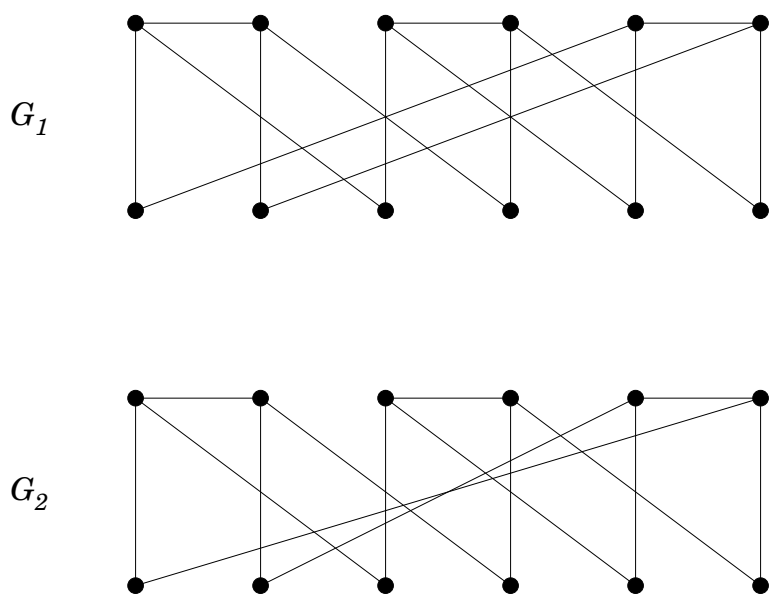

Figure 39: graphs arising from $H=K_{2} \cup K_{2} \cup K_{2}$ in Proposition 3.15
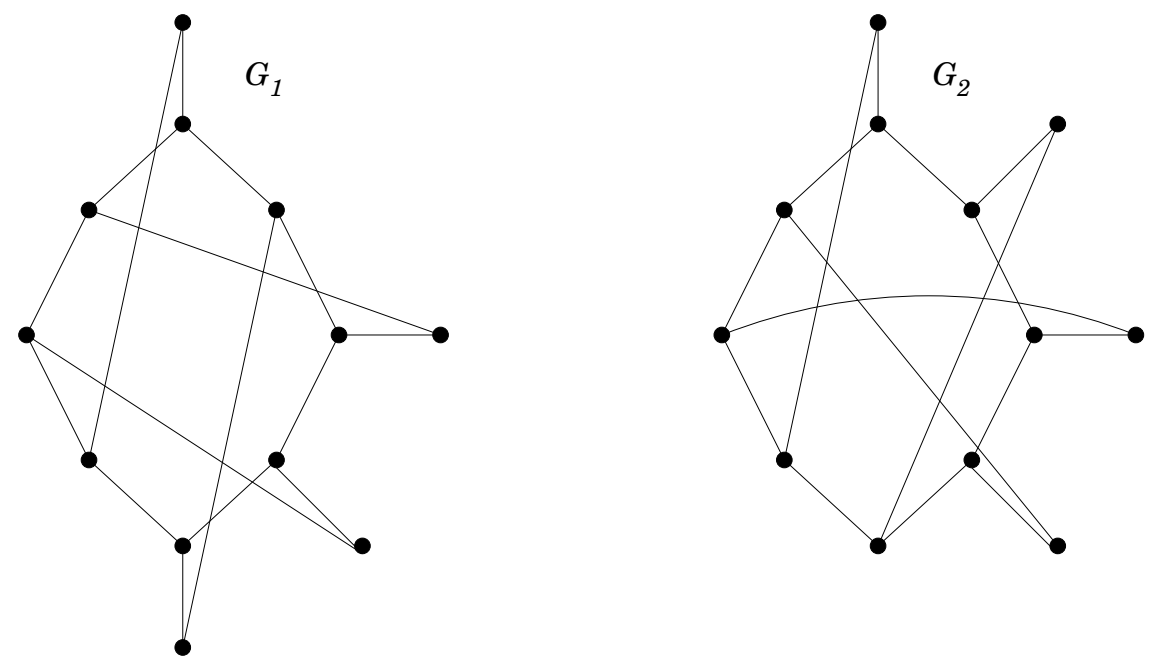

Figure 40: graphs arising from $H=C_{8}$ in Proposition 3.15 

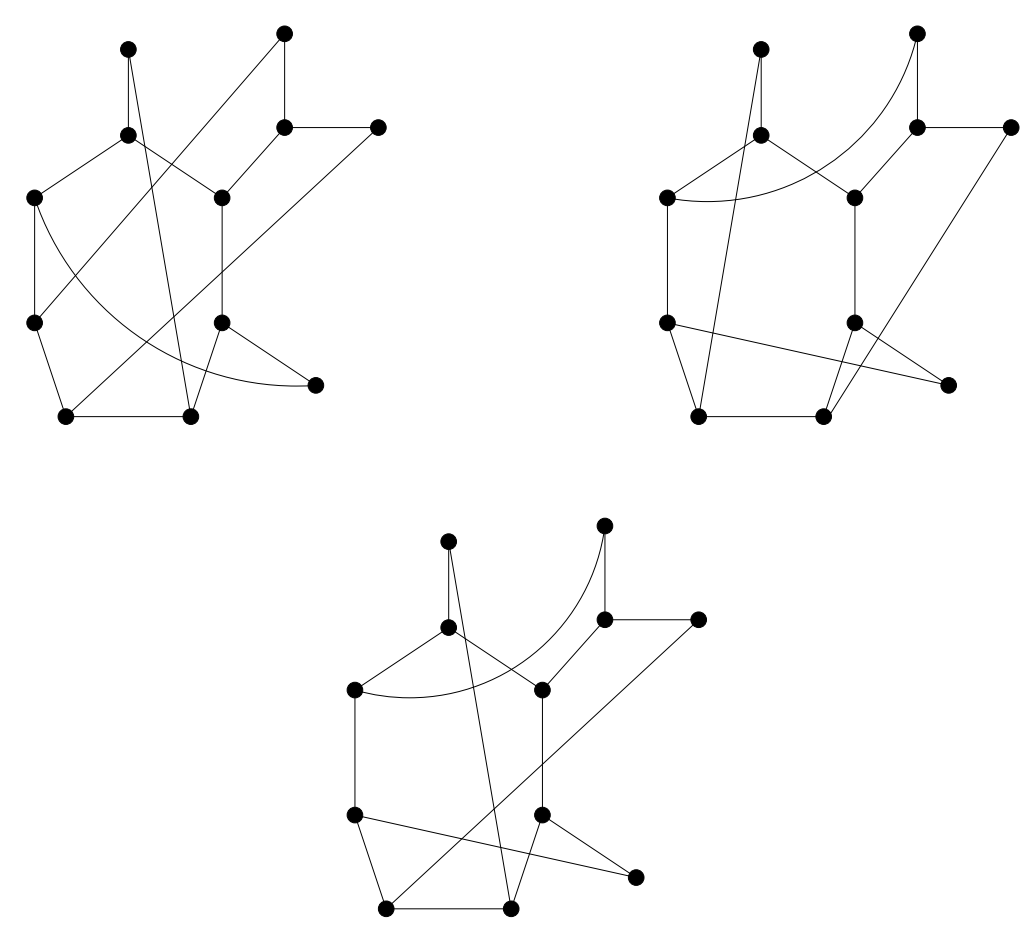

Figure 41: graphs arising if $H$ contains a $C_{7}$ in Proposition 3.15

The graph $H_{5}$ can be completed to the graph shown in Figure $44, H_{6}$ can be completed to the graph shown in Figure 45 , and $H_{7}$ can be completed to one of the graphs shown in Figure 46. None of the graphs in Figures 44-46 is Laplacian integral.

Proposition 3.16 Suppose that $G \in \mathcal{G}$ has $n$ vertices in total, and a vertices of degree 2. Suppose further that $G$ has one 3-cycle, and that each vertex of that 3-cycle has degree 3. Suppose further that $G$ has c 4-cycles and at most one pair of vertices of degree 2 that are adjacent. Then $G \notin \Gamma_{5}$.

Proof: Let $L=D-A$ be the Laplacian matrix for $G$. From Corollary 3.14, we find that $\operatorname{tr}\left(L^{4}\right)=-4 \operatorname{tr}\left(D A^{3}\right)+258 n-192 a+8 c+\delta$, where $\delta$ is 4 or 0 according as there is, or is not, a pair of vertices of degree 2 that are adjacent. Note also that $\operatorname{tr}\left(D A^{3}\right)=18$ (since each vertex on the 3-cycle has degree 3), and from Lemma 3.12, that $\operatorname{tr}\left(L^{3}\right)=54 n-34 a-6$. 


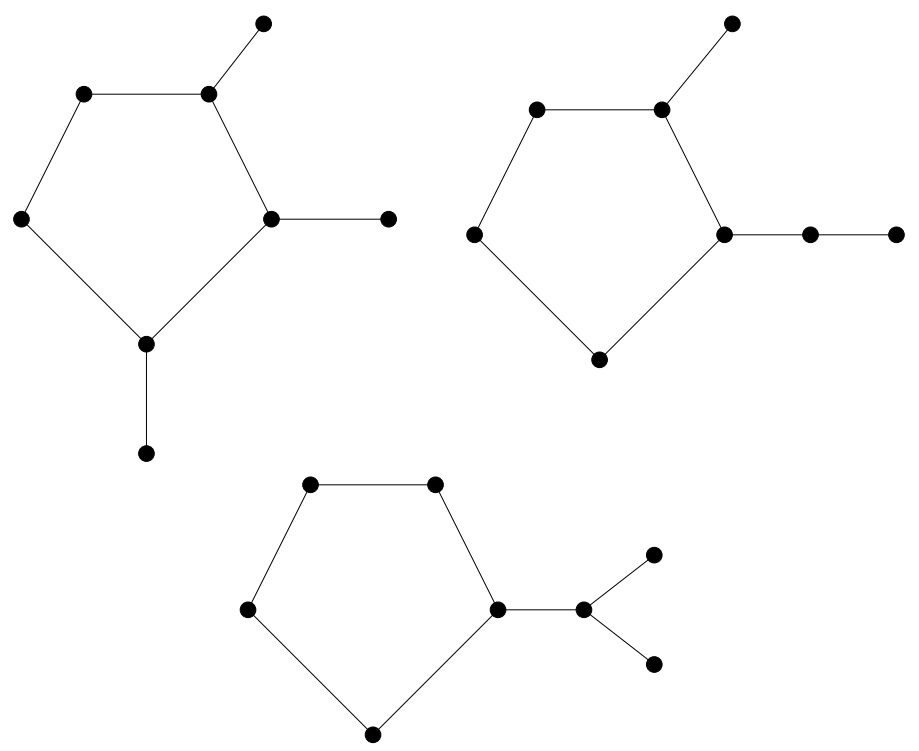

Figure 42: graphs in Proposition 3.15 with $\lambda_{\text {min }}<-2$
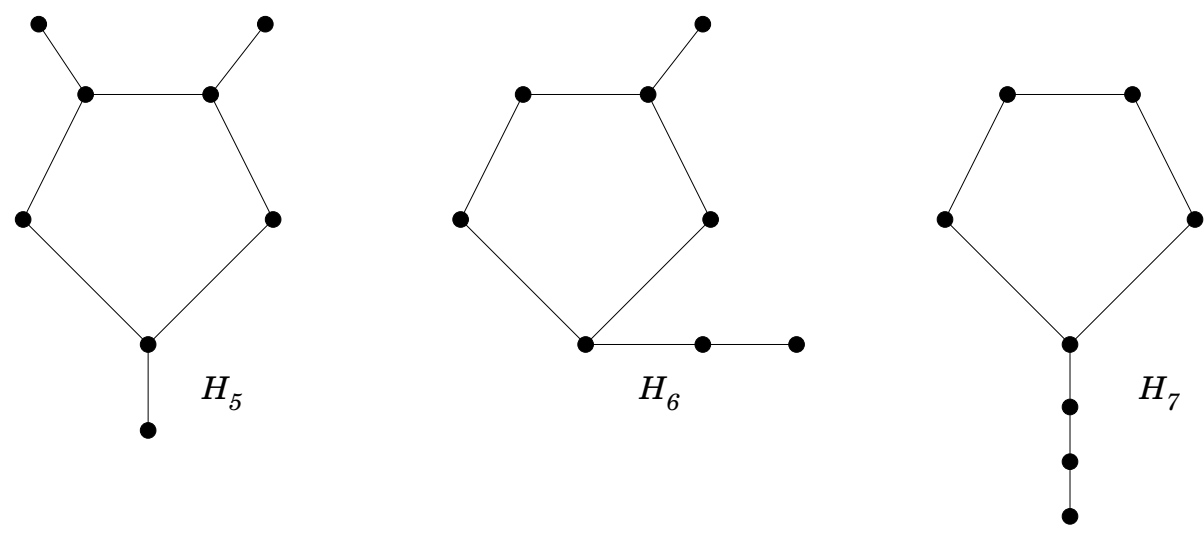

Figure 43: choices for $H$ when it contains a $C_{5}$ in Proposition 3.15 


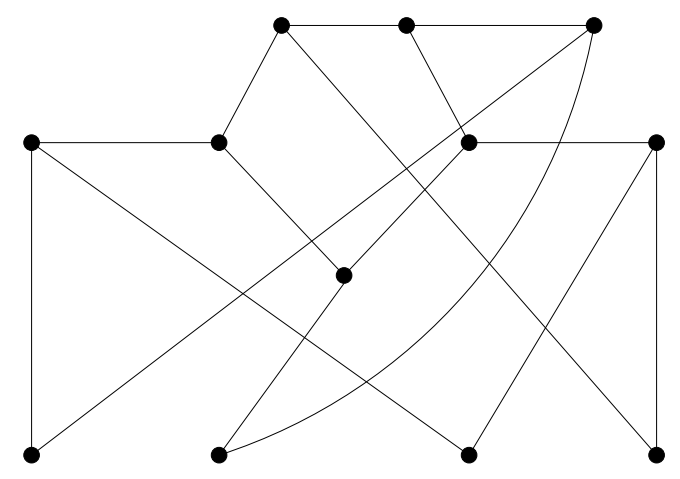

Figure 44: graph arising from $H_{5}$ in Proposition 3.15

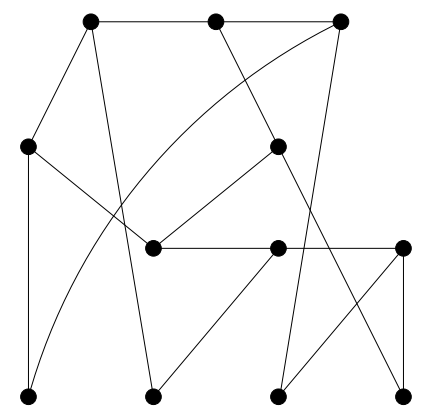

Figure 45: graph arising from $H_{6}$ in Proposition 3.15 

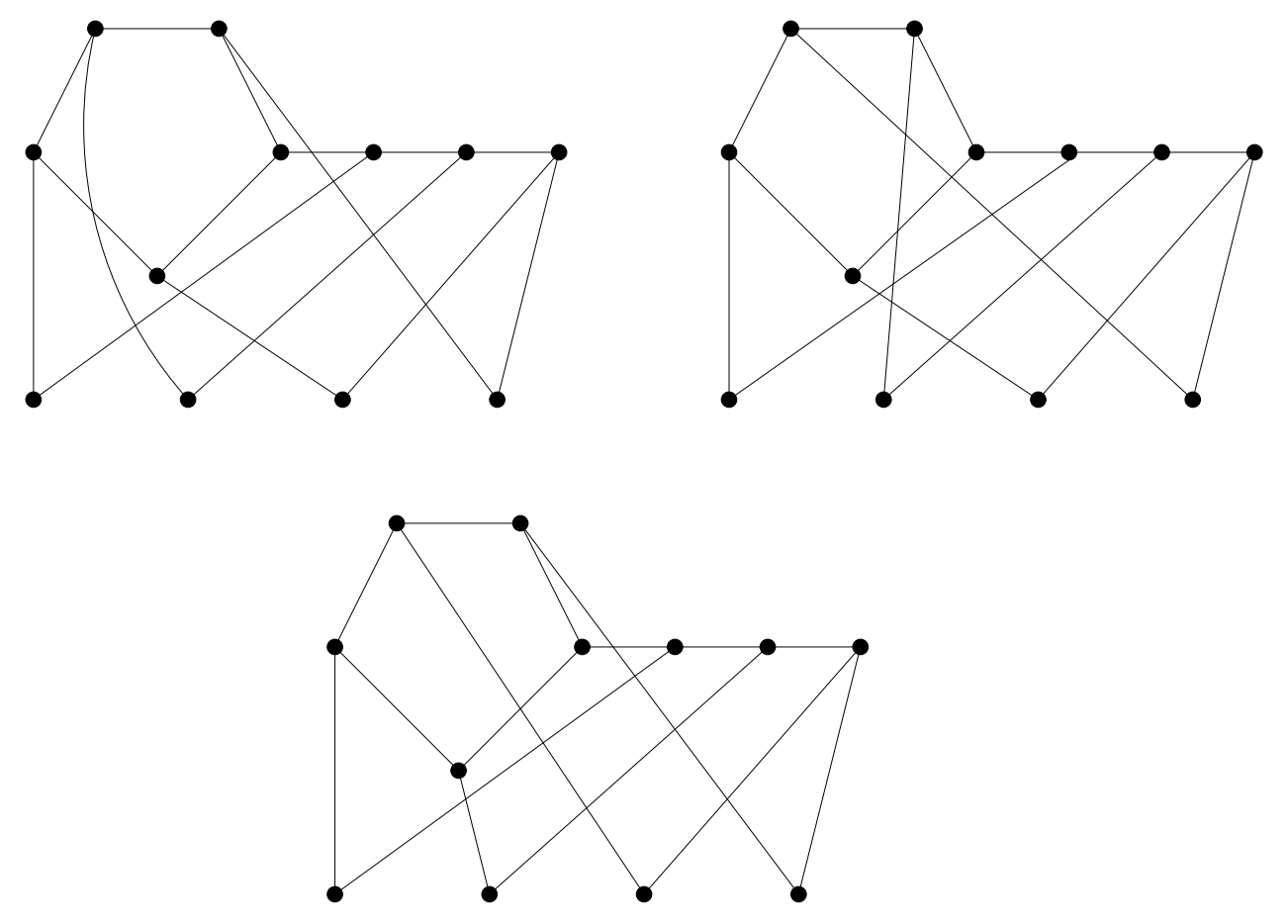

Figure 46: graphs arising from $H_{7}$ in Proposition 3.15

Suppose that in fact $G \in \Gamma_{5}$, and let $m_{1}, \ldots, m_{5}$ be the multiplicities of the eigenvalues $1, \ldots, 5$, respectively. As in Theorem 3.15, it follows that

$$
\left[\begin{array}{l}
m_{1} \\
m_{2} \\
m_{3} \\
m_{4} \\
m_{5}
\end{array}\right]=n\left[\begin{array}{c}
\frac{1}{2} \\
-\frac{1}{2} \\
1 \\
-\frac{1}{2} \\
\frac{1}{2}
\end{array}\right]-a\left[\begin{array}{c}
-\frac{1}{2} \\
\frac{1}{2} \\
0 \\
-\frac{1}{2} \\
\frac{1}{2}
\end{array}\right]+c\left[\begin{array}{c}
\frac{1}{3} \\
-\frac{4}{3} \\
2 \\
-\frac{4}{3} \\
\frac{1}{3}
\end{array}\right]+\left[\begin{array}{c}
-5 \\
10 \\
-10 \\
5 \\
-1
\end{array}\right]+(\delta-72)\left[\begin{array}{c}
\frac{1}{24} \\
-\frac{1}{6} \\
\frac{1}{4} \\
-\frac{1}{6} \\
\frac{1}{24}
\end{array}\right]+\left[\begin{array}{c}
\frac{7}{2} \\
-13 \\
18 \\
-11 \\
\frac{5}{2}
\end{array}\right] .
$$

Note that if $\delta=0$, then considering $m_{1}$, we find that $\frac{c}{3}+\frac{1}{2}$ is an integer, a contradiction. On the other hand, if $\delta=4$, then $\frac{c}{3}+\frac{1}{2}+\frac{1}{6}$ is an integer, so that necessarily $c \equiv 1 \bmod 3$. If $c \geq 4$, we find by considering $m_{2}$ that $0 \leq m_{2} \leq-\frac{n+a}{2}+3$, so that $n+a \leq 6$, a contradiction.

It remains to consider the case that $c=1$ and $\delta=4$ - i.e. that $G$ has one 4 -cycle and one pair of adjacent vertices of degree 2. Substituting the values $c=1, \delta=4$ into (3.5), we find that $m_{3}=n-7$ and that $m_{2}+m_{4}=11-n$, so that necessarily $7 \leq n \leq 11$. We conclude then that either $n=8$ or $n=10$.

Suppose first that the 3-cycle and the 4-cycle share a vertex. Then without loss of generality, $G$ contains the subgraph shown in Figure 47. Note that if vertices 1 and 2 both have degree 2 , then vertex 5 is a cut-vertex, a contradiction. We thus conclude that 
neither member of the pair of adjacent vertices of degree 2 is found among $1, \ldots, 5$; we label that pair 6,7. Applying Lemma 3.1 to the subgraph induced by 6 and 7 first with the 3-cycle, and then with the 4-cycle, it follows that without loss of generality, $G$ contains the edges $5 \sim 7$ and $6 \sim 2$. But then 1 is a cut vertex, a contradiction.

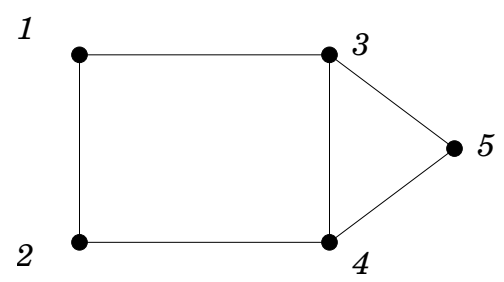

Figure 47: subgraph when the cycles share a vertex in Proposition 3.16

We suppose henceforth that the 3-cycle and the 4-cycle are vertex disjoint. If there is no edge between those two cycles, we find from Lemma 3.1 that $G \notin \mathcal{G}$, a contradiction. Hence there is at least one edge between those cycles.

If there are two such edges, then without loss of generality we have the graph shown in Figure 48 as an induced subgraph of $G$. Necessarily each member of the adjacent pair of vertices of degree 2 falls outside of $1, \ldots, 7$, and applying Lemma 3.1 as above, without loss of generality, we have the subgraph of $G$ that is depicted in Figure 49. In that case, $G$ has a cutpoint, a contradiction.

Next, suppose that there is just one edge between the 3-cycle and the 4-cycle. Note that if $n=8$, two of the vertices on the 3-cycle must be adjacent to a common vertex off of the 3-cycle, which implies the $G$ has more than one 4-cycle, a contradiction. Hence $n=10$. If there are two adjacent vertices of degree 2 on the 4 -cycle, it follows that the only graph satisfying all of the constraints is the one shown in Figure 50, which is not Laplacian integral.

If there are no adjacent vertices of degree 2 on the 4-cycle, we find that neither member of the pair of adjacent vertices of degree 2 is on the 4-cycle. It follows that without loss of generality, $G$ contains the subgraph shown in Figure 51. Applying the constraints on

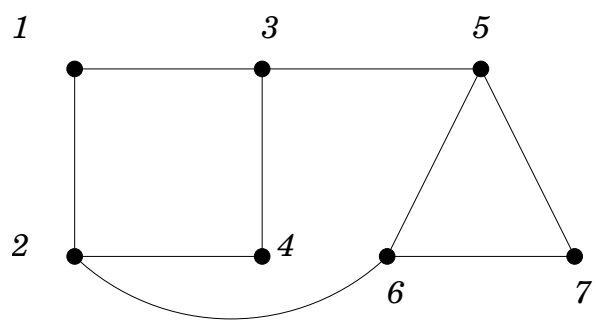

Figure 48: induced subgraph with two edges between the cycles in Proposition 3.16 


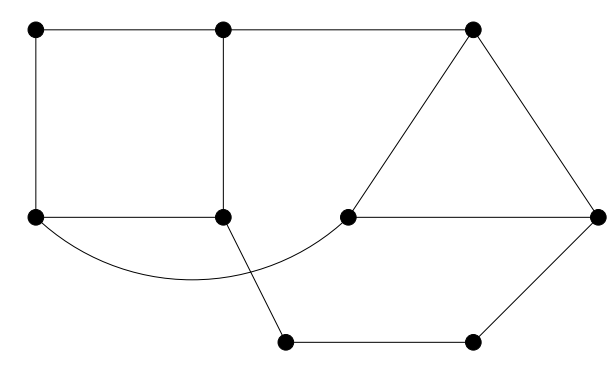

Figure 49: subgraph giving rise to a cutpoint in Proposition 3.16

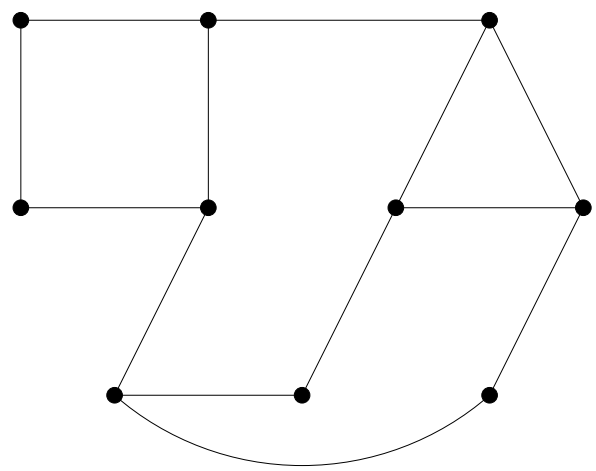

Figure 50: graph for $n=10$ with adjacent vertices of degree 2 on the 4-cycle

$G$, we find that $G$ must be one of the four graphs shown in Figure 52, none of which is Laplacian integral.

We conclude that $G \notin \Gamma_{5}$.

Proposition 3.17 Suppose that $G \in \mathcal{G}$ has $n \geq 10$ vertices in total, and a vertices of degree 2. Suppose further that $G$ has two 3-cycles, and that there is one edge between them. Suppose also that $G$ has at most one pair of vertices of degree 2 that are adjacent. Then $G \notin \Gamma_{5}$.

Proof: Without loss of generality, we have the graph shown in Figure 53 as a subgraph of $G$.

Considering the cut arising from these vertices, it follows that at most one vertex in that subgraph has degree 2 in $G$; we take that vertex (if it exists) to be vertex 1 , of degree $d_{1}$. Letting $L=D-A$ denote the Laplacian matrix for $G$, we have $\operatorname{tr}\left(D A^{3}\right)=2 d_{1}+30$, so that $\operatorname{tr}\left(L^{4}\right)=258 n-192 a+8 c+\delta-120-8 d_{1}$, where $c$ is the number of 4-cycles in $G$ and $\delta$ is either 4 or 0 according as there is, or is not, a pair of adjacent vertices of degree 2 in $G$. Also, note that $\operatorname{tr}\left(L^{3}\right)=54 n-34 a-12$. 


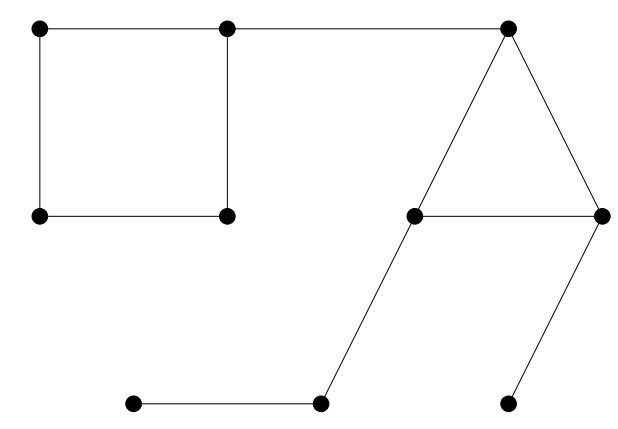

Figure 51: subgraph when there are no adjacent vertices of degree 2 on the 4-cycle

Suppose to the contrary that $G \in \Gamma_{5}$ and let $m_{1}, \ldots, m_{5}$ denote the multiplicities of $1, \ldots, 5$ as eigenvalues of $L$, respectively. As in Theorem 3.15, we have

$$
\left[\begin{array}{l}
m_{1} \\
m_{2} \\
m_{3} \\
m_{4} \\
m_{5}
\end{array}\right]=n\left[\begin{array}{c}
\frac{1}{2} \\
-\frac{1}{2} \\
1 \\
-\frac{1}{2} \\
\frac{1}{2}
\end{array}\right]-a\left[\begin{array}{c}
-\frac{1}{2} \\
\frac{1}{2} \\
0 \\
-\frac{1}{2} \\
\frac{1}{2}
\end{array}\right]+c\left[\begin{array}{c}
\frac{1}{3} \\
-\frac{4}{3} \\
2 \\
-\frac{4}{3} \\
\frac{1}{3}
\end{array}\right]+\left[\begin{array}{c}
-5 \\
10 \\
-10 \\
5 \\
-1
\end{array}\right]+\left(120+8 d_{1}-\delta\right)\left[\begin{array}{c}
-\frac{1}{24} \\
\frac{1}{6} \\
-\frac{1}{4} \\
\frac{1}{6} \\
-\frac{1}{24}
\end{array}\right]+\left[\begin{array}{c}
7 \\
-26 \\
36 \\
-22 \\
5
\end{array}\right] .
$$

Considering $m_{2}+m_{5}$, we see that $\frac{120-8 d_{1}-\delta}{8}$ must be an integer, so that necessarily, $\delta=0$ - i.e. there are no pairs of adjacent vertices of degree 2 . Now considering $m_{1}$, we find that $\frac{c-d_{1}}{3}$ is an integer. From the fact that $m_{2} \geq 0$, it follows that $c-d_{1} \leq 3-\frac{3(n+a)}{8}$. Now $n+a \geq 12$ (recall that $n$ and $a$ have the same parity), and hence $c-d_{1} \leq-\frac{3}{2}$, which yields $c \leq d_{1}-2$ since $c$ and $d_{1}$ are integers. If $d_{1}=2$, then $c=0$, contradicting the fact that $c \equiv d_{1} \bmod 3$, so the only possible case is that $d_{1}=3$ and $c=0$. Since $m_{2} \geq 0$, we have $n+a \leq 16$, and hence $n$ is one of 10,12 and 15 .

If $n=15$, then necessarily $a=1$, and we have

$$
\left[\begin{array}{l}
m_{1} \\
m_{2} \\
m_{3} \\
m_{4} \\
m_{5}
\end{array}\right]=\left[\begin{array}{l}
4 \\
0 \\
5 \\
0 \\
5
\end{array}\right]
$$

From the Cayley-Hamilton theorem, we have $(L-I)(L-3 I)(L-5 I)=-J$, or equivalently, $L^{3}-9 L^{2}+23 L-15 I+J=0$. Consider a vertex $i$ of degree $d_{i}$. It is straightforward to determine that if vertex $i$ is not on a 3 -cycle, then the $i$-th diagonal entries of $L^{3}, L^{2}$ and $L$ are $d_{i}^{3}+2 d_{i}^{2}+\sum_{j \sim i} d_{j}, d_{i}^{2}+d_{i}$, and $d_{i}$, respectively. Considering a vertex $i$ of degree 3 that is adjacent only to other vertices of degree 3 and is not on a 3 -cycle (there must be such a vertex), it follows that the $i$-th diagonal entry of $L^{3}-9 L^{2}+23 L-15 I+J$ must be odd, a contradiction. 

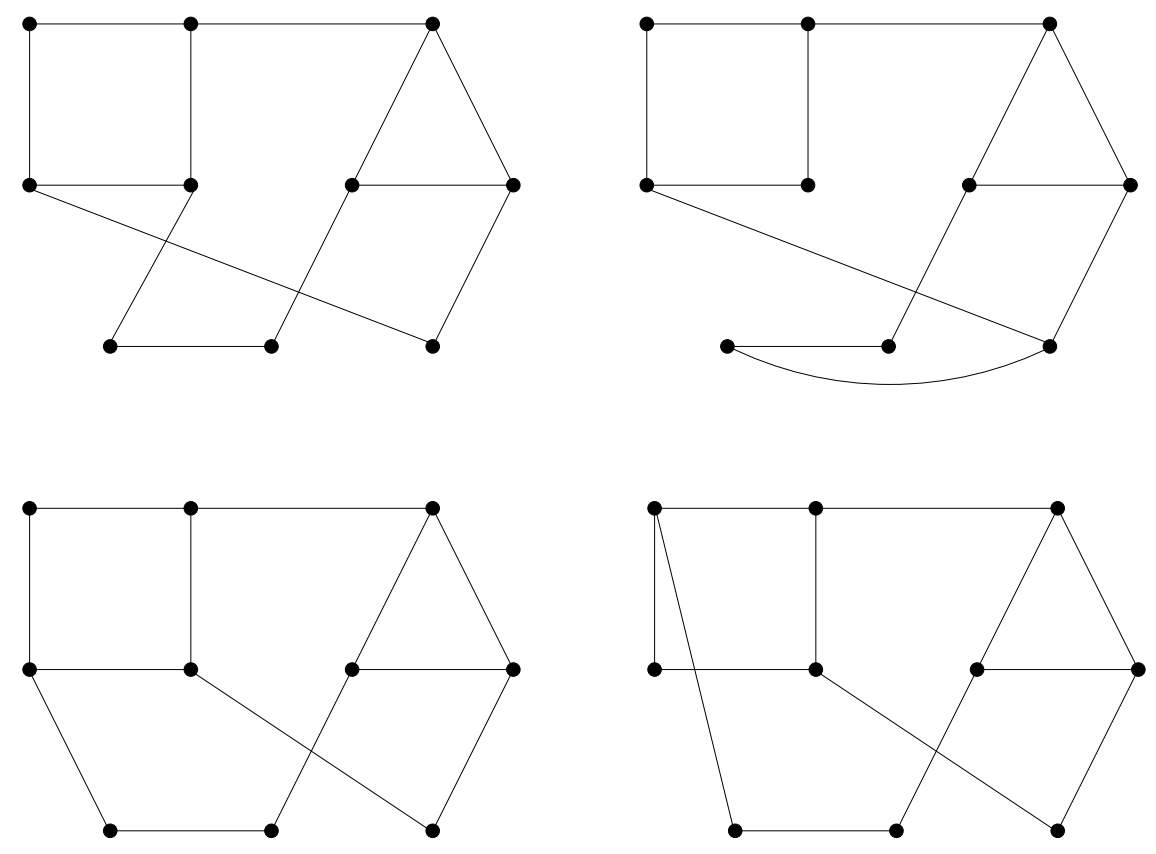

Figure 52: choices for $G$ in Proposition 3.16

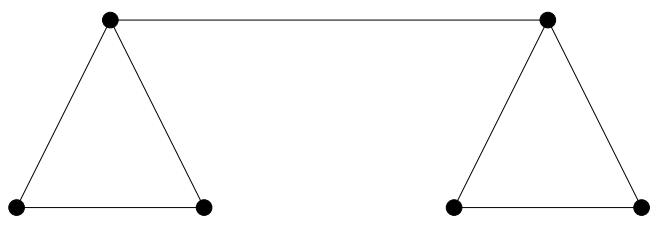

Figure 53: subgraph in Proposition 3.17

Suppose now that $n$ is either 10 or 12 . Applying the above constraints on $G$ - i.e. $d_{1}=3, c=0, \delta=0$, along with the hypotheses on $G$ - it follows that without loss of generality $G$ contains one of the graphs $G_{1}, G_{2}, G_{3}$ shown in Figure 54 as a subgraph.

If $G_{3}$ is a subgraph, then considering the cut in $G$ arising from the vertices of $G_{3}$, we find that $n \leq 10$, so that in fact $n=10$. It follows readily that the only graph on 10 vertices containing $G_{3}$ with the required degree sequence is the one in Figure 55. That graph has an adjacent pair of vertices of degree 2, a contradiction; we conclude that $G_{3}$ can not be a subgraph of $G$. Suppose that $n=10$, so that $G$ contains either $G_{1}$ or $G_{2}$. In the former case, $G$ must be the graph shown in Figure 56, which is not Laplacian integral; in the latter case, $G$ is one of the graphs in Figure 57, none of which is Laplacian integral. Thus $n=10$ leads to a contradiction in all possible cases. 

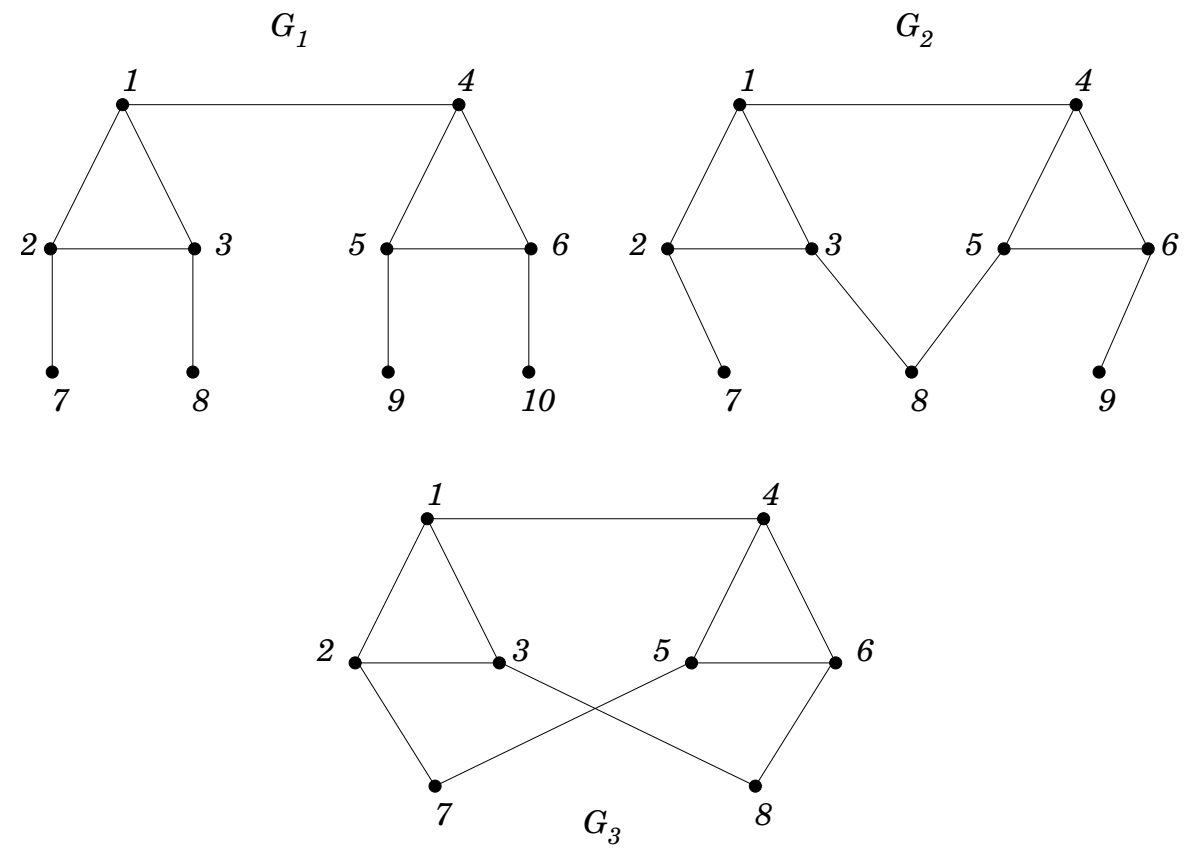

Figure 54: the subgraphs $G_{1}, G_{2}, G_{3}$ in Proposition 3.17

Henceforth, we consider the case that $n=12$. Recall that $n+a \leq 16$, so that either $a=2$ or $a=4$. For $a=2$ we have

$$
\left[\begin{array}{l}
m_{1} \\
m_{2} \\
m_{3} \\
m_{4} \\
m_{5}
\end{array}\right]=\left[\begin{array}{l}
3 \\
1 \\
2 \\
2 \\
3
\end{array}\right],
$$

while for $a=4$ we have

$$
\left[\begin{array}{l}
m_{1} \\
m_{2} \\
m_{3} \\
m_{4} \\
m_{5}
\end{array}\right]=\left[\begin{array}{l}
4 \\
0 \\
2 \\
3 \\
2
\end{array}\right] .
$$

Suppose that $a=4$; it follows from the Cayley-Hamilton theorem that $L^{4}-13 L^{3}+$ $59 L^{2}-107 L+60 I=5 J$. Consider a vertex $i$ of degree $d_{i}$ that is not on a 3 -cycle. We find that the $i$-th diagonal entries of $L^{4}, L^{3}, L^{2}$ and $L$ are $d_{i}^{4}+3 d_{i}^{3}+2 d_{i} \sum_{j \sim i} d_{j}+$ $\sum_{j \sim i} d_{j}^{2}+d_{i}^{2}+\sum_{j \sim i}\left(d_{j}-1\right), d_{i}^{3}+2 d_{i}^{2}+\sum_{j \sim i} d_{j}, d_{i}^{2}+d_{i}$, and $d_{i}$, respectively. In particular, if $i$ has degree 2 , it is not on any 3-cycle, and we find that the $i$-th diagonal entry of $L^{4}-13 L^{3}+59 L^{2}-107 L+60 I$ must be even, a contradiction to the equation $L^{4}-13 L^{3}+$ $59 L^{2}-107 L+60 I=5 J$. 


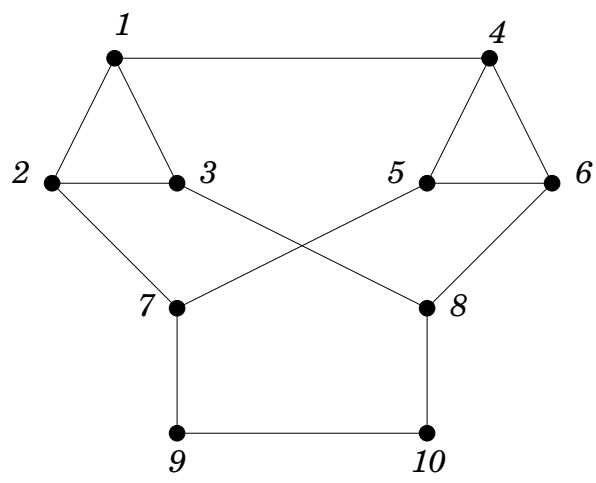

Figure 55: graph arising if $G_{3}$ is a subgraph in Proposition 3.17

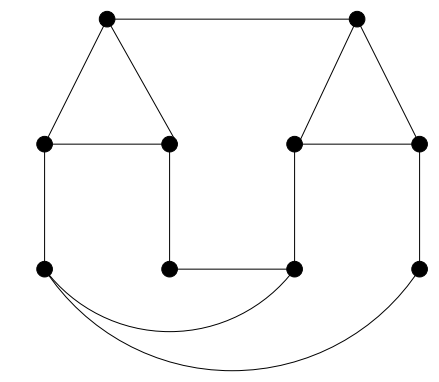

Figure 56: graph arising from $G_{1}$ in Proposition 3.17 when $n=10$

It remains to consider the case that $n=12$ and $a=2$. There are two possible cases: $G_{1}$ is a subgraph of $G$, and $G_{2}$ is a subgraph of $G$. Suppose first that $G_{2}$ is a subgraph of $G$, say on vertices $1, \ldots, 9$ (see Figure 54). It follows that the subgraph $S$ induced by vertices $7, \ldots, 12$ must have six edges. Since $S$ has no cycles of length 3 or 4 , and since the degree of vertex 8 (as a vertex in $S$ ) is at most one, it follows that $S$ consists of a 5-cycle with the pendant vertex 8 appended. Without loss of generality, we take $8 \sim 11$. Since the degrees of vertices 10 and 12 are 2 in $G$, those two vertices are not adjacent. Since $G$ has no 4-cycles, it cannot be the case that we have both $10 \sim 7,9$ and $12 \sim 7,9$, so without loss of generality, we assume that $10 \sim 7$ and $10 \sim 11$. Applying the constraints on $G$ yields one of the two candidate graphs in Figure 58; neither of those graphs is Laplacian integral, a contradiction.

Our final case is that $G_{1}$ is a subgraph of $G$. Then $G$ has 17 edges, and contains $G_{1}$ as a subgraph. Thus the subgraph $S$ on vertices $7, \ldots, 12$ has six edges, and so contains a cycle. Since $S$ contains no 3 -cycle or 4 -cycle, it follows that $S$ is either a 6 -cycle, or $S$ consists of a 5 -cycle with a pendant edge attached. Since $m_{1}=3$, it follows that the principal submatrix of $L$ on rows and columns $1, \ldots, 10, L([1, \ldots, 10])$ say, has 1 as an eigenvalue. If $S$ consists of a 5 -cycle with a pendant edge attached, we take the pendant 

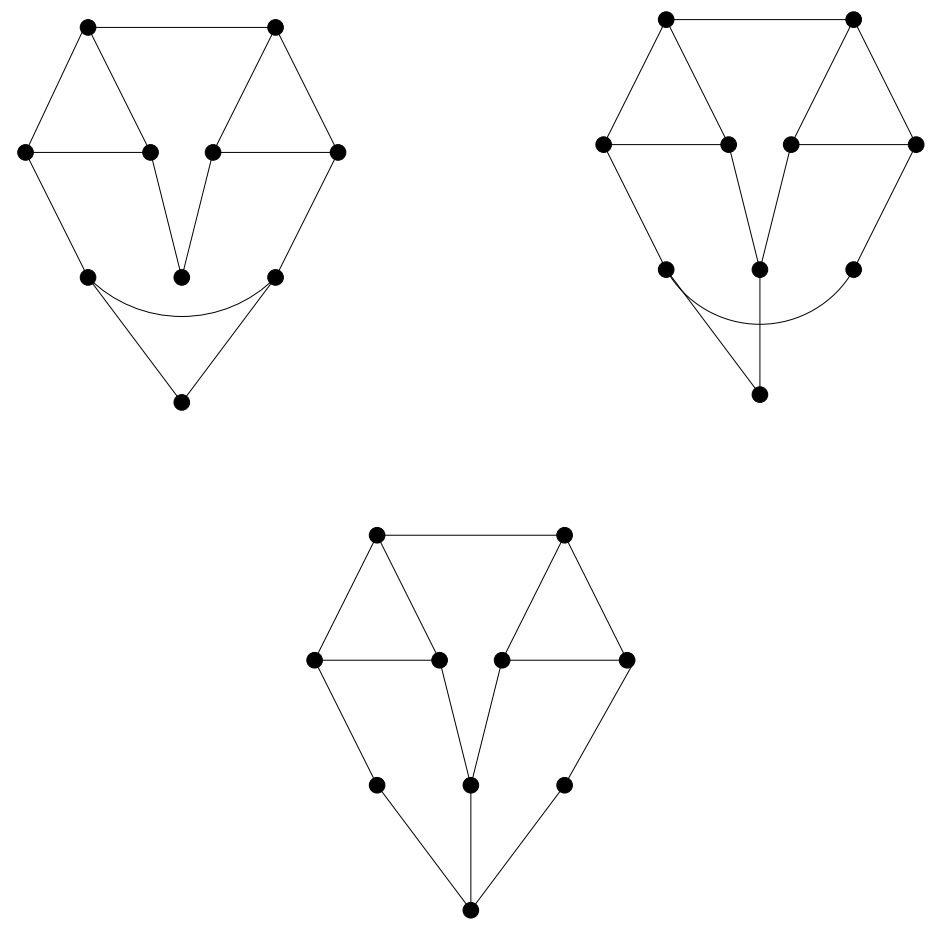

Figure 57: graphs arising from $G_{2}$ in Proposition 3.17 when $n=10$

vertex of $S$ to be 10, and the vertex of degree 3 to be 12, without loss of generality. Note that each of vertices 7,8,9 has degree 3 in $G$ while 10 has degree 2 . There are either one or two edges between the vertices $7,8,9$,; note that 7 is not adjacent to 8 , otherwise $G$ has a 4 -cycle. We are then led to three possibilities for $L([1, \ldots, 10])$, none of which has 1 as an eigenvalue. Suppose finally that $S$ is a 6 -cycle, so that each of vertices $7,8,9,10$ has degree 3 in $G$, with vertices 11 and 12 not adjacent (since $\delta=0$ ). It follows that there are exactly two edges between vertices in $\{7,8,9,10\}$; without loss of generality, we have either $7 \sim 10,8 \sim 9$ or $7 \sim 9,8 \sim 9$. The latter case cannot occur though, since then $L([1, \ldots, 10])$ does not have 1 as an eigenvalue. It follows that, without loss of generality, $G$ is one of the graphs shown in Figure 59. As those graphs are not Laplacian integral, we obtain a final contradiction.

We are at last ready to prove the main result, which is restated below.

Theorem 3.18 Let $G$ be a connected non-regular graph such that $\Delta(G)=3$. Then $G$ is Laplacian integral if and only if it is one of the graphs depicted in Figures 4 and 5.

Proof: It is straightforward to determine that each of the graphs in Figures 4 and 5 is Laplacian integral. 

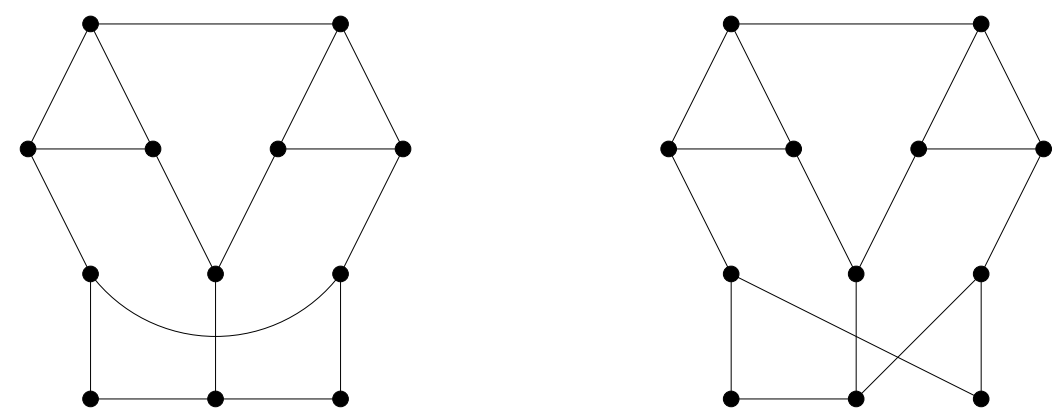

Figure 58: graphs arising from $G_{2}$ in Proposition 3.17 when $n=12$
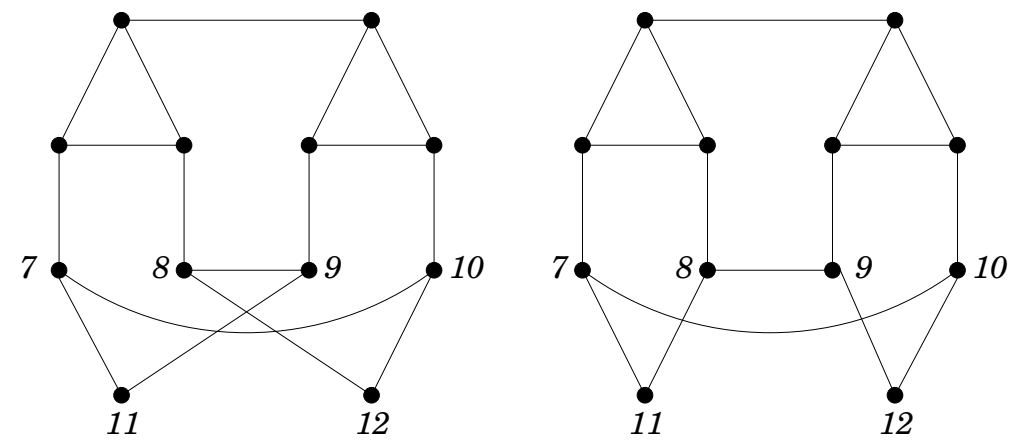

Figure 59: graphs arising from $G_{1}$ in Proposition 3.17 when $n=12$

Suppose now that $G$ is a non-regular graph, that $\Delta(G)=3$, and that $G$ is Laplacian integral. If $G$ has two or more pairs of adjacent vertices of degree 2 , then by Lemma 3.5, there must be two independent edges for which all four end points have degree 2 ; in that case, by Lemma 3.4, $G$ is the graph $D_{1}$ in Figure 4 . Henceforth, we may assume that $G$ has at most one adjacent pair of vertices of degree 2 .

Suppose now that $G$ contains a 3-cycle. By Lemma 3.10 and Proposition 3.16, we find that $G$ must contain at least two 3-cycles, while by Lemma 3.6, we see that every pair of 3-cycles in $G$ must be vertex disjoint. Further, by Lemma 3.9, Remark 3.11 and Proposition 3.17, it cannot be the case that between every pair of 3-cycles there is exactly one edge. Hence, there is a pair of 3-cycles in $G$ with two edges between them, or there is a pair of 3-cycles in $G$ with no edges between them. In the former case, Lemma 3.8 applies and we find that $G$ is the graph $D_{2}$ in Figure 4. In the latter case, Lemma 3.7 applies, and hence $G$ is the graph $D_{3}$ in Figure 4 .

Finally, if $G$ is 3-cycle-free, then Proposition 3.15 applies, and it follows that $G$ is one of the graphs $D_{4}, \ldots, D_{9}$ in Figures 5 . 


\section{Commentary and consequences}

It is straightforward to see that if an edge is added into a graph, then none of the corresponding Laplacian eigenvalues can decrease, and that the sum of the Laplacian eigenvalues must increase by 2 . In the special case that the addition of an edge results in the Laplacian eigenvalues changing only by integer quantities, we find that just two situations can arise: either one eigenvalue increases by 2 , or two eigenvalues increase by 1 each. These situations are known as spectral integral variation in one and two places, respectively. A characterization of the former is given in [22], while the latter is characterized in [13].

The following remark identifies some relationships between a few of the graphs that appear in Figures 3, 4, and 5.

Remark 4.1 We have the following observations.

1. The graph $D_{1}$ in Figure 4 is formed from $C_{6}$ by adding an edge between vertices at distance 3. Thus, spectral integral variation in two places occurs under that edge addition, with the eigenvalues 1 and 4 of $C_{6}$ increasing to 2 and 5 , respectively.

2. The graph $D_{2}$ in Figure 4 is the complement of $D_{1}$.

3. Note that the graph $A_{2}$ in Figure 3 in can be constructed from $D_{3}$ in Figure 4 by the addition of a single edge (between the vertices of degree 2 in $D_{3}$ ); that edge addition causes the eigenvalues 1 and 4 of $D_{3}$ to increase to 2 and 5 , respectively, in $A_{2}$.

4. In Figure 60, we have drawn the graphs $D_{5}$ and $D_{6}$ in a somewhat more suggestive manner, so as to emphasize their relationships with the graphs $D_{7}$ and $A_{1}$. Inspecting Figure 60 we see that $D_{6}$ can be constructed from $D_{5}$ by the addition of a single edge, with the eigenvalues 1 and 4 increasing to 2 and 5, respectively. Further, $D_{7}$ can be constructed from $D_{6}$ by the addition of a single edge, again with the eigenvalues 1 and 4 increasing to 2 and 5 , respectively. Finally, note that $A_{1}$ can be constructed from $D_{7}$ by the addition of a single edge, also with the eigenvalues 1 and 4 increasing to 2 and 5 , respectively.

5. Looking at the spectra and degree sequences of the graphs $D_{8}$ and $D_{9}$, it is natural to wonder whether $D_{9}$ can be constructed from $D_{8}$ by the addition of a single edge (with the effect that the eigenvalues 1 and 4 would increase to 2 and 5). However, that turns out not to be the case. For example, it is straightforward to see that there is no way to add an edge between vertices of degree 2 in $D_{8}$ so that the vertices of degree 3 in the resulting graph then induce an 8-cycle.

In [5] the authors conjecture that there is no connected graph on $n$ vertices having Laplacian spectrum $0,1,2, \ldots, n-2, n-1$. Tangentially related to that conjecture is the question of whether, for a given $k \in \mathbb{N}$, there exists a connected graph whose distinct Laplacian eigenvalues are $0,1,2, \ldots, k$. The following result deals with that question. 

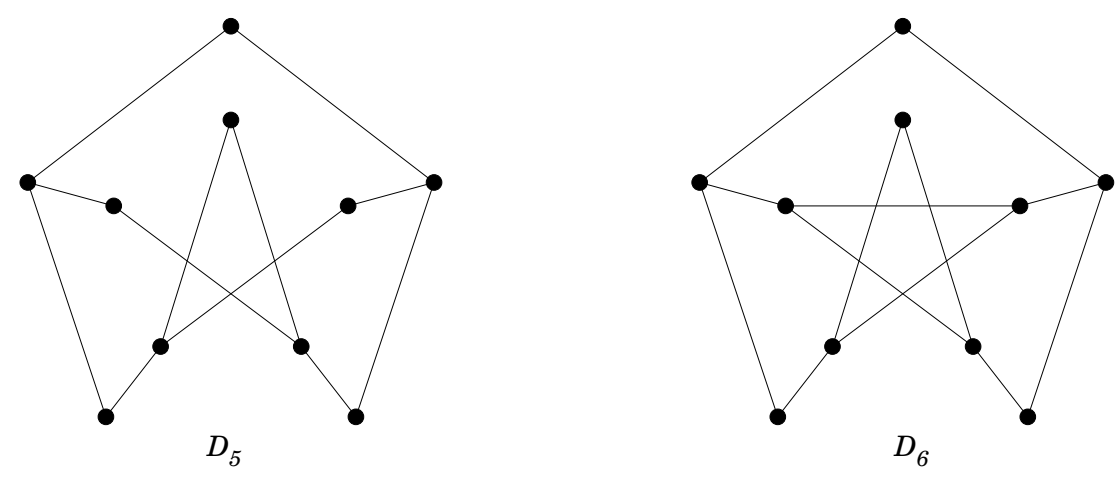

Figure 60: alternative drawings of $D_{5}$ and $D_{6}$

Theorem 4.2 Let $k \in \mathbb{N}$. There is a connected graph $G$ whose distinct Laplacian eigenvalues are $0,1,2, \ldots, k-1, k$ if and only if $k \geq 5$.

Proof: Suppose first that for some integer $k$ with $1 \leq k \leq 4$, there is a connected graph $G$ on $n$ vertices with distinct Laplacian eigenvalues $0,1,2, \ldots, k$. Then $\Delta(G)+1 \leq k$, so that in particular, $\Delta(G) \leq 3$. Note that $\Delta(G)=3$ only if $n=k=4$. Evidently there is no graph on 4 vertices with the five Laplacian eigenvalues $0,1,2,3,4$. Hence we must have $\Delta(G) \leq 2$; as noted in Section 1, there are exactly five Laplacian integral graphs with maximum degree at most 2 ; none of them has the required spectrum. Thus, if there is a connected graph with distinct Laplacian eigenvalues $0,1,2, \ldots, k-1, k$, it must be the case that $k \geq 5$.

From Theorem 2.1 and Figure 5, the graphs $D_{8}$ and $D_{9}$ have distinct Laplacian eigenvalues $0,1,2,3,4$ and 5 . Next, consider the graph $H$ formed by adding a pendant edge to a 3-cycle; $H$ has spectrum $0,1,3,4$. The Cartesian product (see [7]) of $H$ with $K_{2}$ then has spectrum $0,1,2,3^{(2)}, 4,5,6$. Thus we see that for $k=5,6$, there is a connected graph whose distinct Laplacian eigenvalues are $0,1,2, \ldots, k-1, k$. Finally, note that for any $k \geq 7$, an induction step shows that there is a connected graph $G$ with Laplacian eigenvalues $0,1,2, \ldots, k-2$; the Cartesian product of $G$ with $K_{2}$ is connected and has Laplacian eigenvalues $0,1,2, \ldots, k$, as desired.

\section{References}

[1] N. Abreu, New and old results on algebraic connectivity of graphs, Linear Algebra and its Applications 423 (2007), 53-73. 
[2] F. Bussemaker and D. Cvetkovic, There are exactly 13 connected, cubic, integral graphs, Univerzitet u Beogradu, Publikacije Elektrotehnickog Fakulteta, Serija Matematika i Fizika 544-576 (1976), 43-48

[3] D. Cvetkovic, Cubic integral graphs, Univerzitet u Beogradu, Publikacije Elektrotehnickog Fakulteta, Serija Matematika i Fizika 498-541 (1975), 107-113.

[4] D. Corneil, H. Lerch and L. Stewart Burlingham, Complement reducible graphs, Discrete Applied Mathematics 3 (1981), 163-174.

[5] S. Fallat, S. Kirkland, J. Molitierno and M. Neumann, On graphs whose Laplacian matrices have distinct integer eigenvalues, Journal of Graph Theory 50 (2005), 162174 .

[6] M. Fiedler, Algebraic connectivity of graphs, Czechoslovak Mathematical Journal 23(98) (1973), 298-305.

[7] C. Godsil and G. Royle, Algebraic Graph Theory, Springer, New York, 2001.

[8] R. Grone and R. Merris, The Laplacian spectrum of a graph II, SIAM Journal on Discrete Mathematics 7 (1994), 221-229.

[9] R. Grone and R. Merris, Indecomposable Laplacian integral graphs, Linear Algebra and its Applications, to appear.

[10] P. Hammer and A. Kelmans, Laplacian spectra and spanning trees in threshold graphs, Discrete Applied Mathematics 65 (1996), 255-273.

[11] F. Harary and A. Schwenk, Which graphs have integral spectra?, Lecture Notes in Mathematics 406 (1974), 45-51.

[12] S. Kirkland, A bound on the algebraic connectivity of a graph in terms of the number of cutpoints, Linear and Multilinear Algebra 47 (2000), 93-103.

[13] S. Kirkland, A characterization of spectral integral variation in two places for Laplacian matrices, Linear and Multilinear Algebra 52 (2004), 79-98.

[14] S. Kirkland, Completion of Laplacian integral graphs via edge addition, Discrete Mathematics 295 (2005), 75-90.

[15] S. Kirkland, Constructably Laplacian Integral Graphs, Linear Algebra and its Applications 423 (2007), 3-21.

[16] S. Kirkland, J. Molitierno, M. Neumann and B. Shader, On Graphs with Equal Algebraic and Vertex Connectivity, Linear Algebra and its Applications 341 (2002), 45-56.

[17] R. Merris, Degree maximal graphs are Laplacian integral, Linear Algebra and its Applications 199 (1994), 381-389.

[18] R. Merris, Large families of Laplacian isospectral graphs, Linear and Multilinear Algebra 43 (1997), 201-205. 
[19] R. Merris, Laplacian matrices of graphs: a survey, Linear Algebra and its Applications 197, 198 (1994), 143-176.

[20] B. Mohar, Laplace eigenvalues of graphs-a survey, Discrete Mathematics 109 (1992), 171-183.

[21] A. Schwenk, Exactly thirteen connected cubic graphs have integral spectra, in Theory and Applications of Graphs, Y. Alavi and D. Lick, eds., Lecture Notes in Mathematics 642 (1978), 516-533.

[22] W. So, Rank one perturbation and its application to the Laplacian spectrum of a graph, Linear and Multilinear Algebra 46 (1999), 193-198. 